\title{
Article \\ Association of Genetic Polymorphisms in Oxidative Stress and Inflammation Pathways with Glaucoma Risk and Phenotype
}

\author{
Makedonka Atanasovska Velkovska ${ }^{1}$ (D), Katja Goričar ${ }^{2}$, Tanja Blagus ${ }^{2}$, Vita Dolžan ${ }^{2}$ (D) and Barbara Cvenkel ${ }^{1,3, *}$ \\ 1 Department of Ophthalmology, University Medical Centre Ljubljana, 1000 Ljubljana, Slovenia; \\ atmakedonka@yahoo.com \\ 2 Pharmacogenetics Laboratory, Institute of Biochemistry and Molecular Genetics, Faculty of Medicine, \\ University of Ljubljana, 1000 Ljubljana, Slovenia; katja.goricar@mf.uni-lj.si (K.G.); \\ tanja.blagus@mf.uni-lj.si (T.B.); vita.dolzan@mf.uni-lj.si (V.D.) \\ 3 Faculty of Medicine, University of Ljubljana, 1000 Ljubljana, Slovenia \\ * Correspondence: barbara.cvenkel@gmail.com; Tel.: +386-(40)-233-462
}

\section{check for} updates

Citation: Atanasovska Velkovska, M.; Goričar, K.; Blagus, T.; Dolžan, V.; Cvenkel, B. Association of Genetic Polymorphisms in Oxidative Stress and Inflammation Pathways with Glaucoma Risk and Phenotype. J. Clin. Med. 2021, 10, 1148. https:// doi.org/10.3390/jcm10051148

Academic Editors: Jose Javier Garcia-Medina and Maria Dolores Pinazo-Duran

Received: 20 January 2021

Accepted: 4 March 2021

Published: 9 March 2021

Publisher's Note: MDPI stays neutral with regard to jurisdictional claims in published maps and institutional affiliations.

Copyright: (c) 2021 by the authors. Licensee MDPI, Basel, Switzerland. This article is an open access article distributed under the terms and conditions of the Creative Commons Attribution (CC BY) license (https:// creativecommons.org/licenses/by/ $4.0 /)$.
Abstract: Oxidative stress and neuroinflammation are involved in the pathogenesis and progression of glaucoma. Our aim was to evaluate the impact of selected single-nucleotide polymorphisms in inflammation and oxidative stress genes on the risk of glaucoma, the patients' clinical characteristics and the glaucoma phenotype. In total, 307 patients with primary open-angle glaucoma or ocular hypertension were enrolled. The control group included 339 healthy Slovenian blood donors. DNA was isolated from peripheral blood. Genotyping was performed for SOD2 rs4880, CAT rs1001179, GPX1 rs1050450, GSTP1 rs1695, GSTM1 gene deletion, GSTT1 gene deletion, IL1B rs1143623, IL1B rs16944, IL6 rs1800795 and TNF rs1800629. We found a nominally significant association of GSTM1 gene deletion with decreased risk of ocular hypertension and a protective role of IL1B rs16944 and IL6 rs1800629 in the risk of glaucoma. The CT and TT genotypes of GPX1 rs1050450 were significantly associated with advanced disease, lower intraocular pressure and a larger vertical cup-disc ratio. In conclusion, genetic variability in IL1B and IL6 may be associated with glaucoma risk, while GPX and TNF may be associated with the glaucoma phenotype. In the future, improved knowledge of these pathways has the potential for new strategies and personalised treatment of glaucoma.

Keywords: glaucoma; inflammation; oxidative stress; phenotype; polymorphism; susceptibility

\section{Introduction}

There is growing interest in the correlation between oxidative stress, inflammation, apoptosis and primary open-angle glaucoma (POAG) initiation and progression [1-5]. Reactive oxygen species (ROS) are formed in the eyes, following a wide variety of stressors and are largely implicated in glaucoma pathogenesis. Similarly, immune-inflammatory response mediators have recently become a target of interest in glaucoma [6-10].

POAG progression has been linked to an increase in oxidative stress (OS) markers [11,12], and although it has been hypothesised that OS plays an early role in the development of glaucomatous optic neuropathy [13], the link between OS and clinical glaucoma parameters remains to be elucidated [1]. In glaucoma, increased intraocular pressure (IOP), vascular dysregulation and immune system activation can trigger several changes in the retina and optic nerve, including disrupted axonal transport and neurofilament accumulation, microvascular abnormalities, extracellular matrix remodelling and glial cell activation. These alterations can lead to secondary damage such as excitotoxicity, neurotrophin deprivation, oxidative damage, mitochondrial dysfunction and, eventually, retinal ganglion cell death $[5,14]$. In addition, neurodegeneration extends beyond the retina and optic nerve into the central nervous system $[3,15]$. 
Oxidative stress arises due to disturbed equilibrium between the pro-oxidant/antio xidant status that further takes part in the generation of ROS and free radicals, both potentially toxic for neuronal cells. The human body produces oxygen free radicals and other ROS as by-products through numerous physiological and biochemical processes [16]. At the same time, antioxidants, further supported with antioxidant enzymes superoxide dismutase (SOD), catalase (CAT), glutathione reductase and glutathione peroxidase (GPX), help to regulate the level of ROS $[17,18]$. Decreased antioxidant defence, together with increasing pro-oxidants in the aqueous humour [12,19], ocular tissues [20,21] and blood [22], has been reported in glaucoma. Several antioxidant enzymes such as SOD, CAT and GPX have been found in the aqueous humour [23,24]. The enzyme CAT is the primary scavenger of ROS, and its deficiency and/or genetic variants are associated with a higher risk of diabetes complications and vitiligo [25,26]. GPX has antioxidant effects and catalyses the reduction of hydrogen peroxide by two molecules of glutathione as part of a ROS defence system.

Glaucoma is a neurodegenerative optic neuropathy and, similarly to other neurodegenerative diseases, is also associated with neuroinflammation where astrocytes and microglia play a major role [27]. The presence of reactive astrocytes, microglial activation and the release of inflammatory mediators such as cytokines, ROS, nitric oxide (NO) and tumour necrosis factor- $\alpha$ (TNF- $\alpha$ ) cause a state of chronic inflammation that may exert neurotoxic effects [28]. Immune inflammatory response mediators like proteolytic enzymes and proinflammatory cytokines (TNF- $\alpha$, IL-1 $\beta$, IL-6, IL-12, and NO) have recently become a target of glaucoma research $[15,29-40]$. TNF- $\alpha$, a major immunomodulator and inflammatory cytokine, has been suggested to mediate the apoptotic death of retinal ganglion cells in glaucoma patients [34]. Interleukin-1, an inflammatory cytokine, is implicated in ischaemic and excitotoxic damage in the retina [41]. Interleukin-6, a proinflammatory cytokine, modulates neuronal survival and protects retinal ganglion cells from ischaemic and excitotoxic damage [42].

Moreover, further research concerning the functions, effectors and signalling pathways of the above molecules and their interactions may lead to new strategies for the treatment of glaucoma $[10,43]$.

Genetic factors, such as single-nucleotide polymorphisms (SNPs), have been shown to modify these pathways, but their impact on the risk of glaucoma and disease course has not been confirmed yet. Studies evaluating the effect of genetic polymorphisms or alterations in proteins' functions in the oxidative-stress- and inflammation-related genes on the risk of glaucoma have found conflicting results [44,45]. Although the association of genetic variability of inflammation and oxidative stress genes with the risk of glaucoma has already been explored to some extent, the association with patients' clinical characteristics and glaucoma phenotype has not been studied yet.

Therefore, the aim of our study was to investigate the associations of selected SNPs in inflammation and oxidative stress pathways with the risk of glaucoma, as well as the associations between selected SNPs, patients' clinical characteristics and the glaucoma phenotype.

\section{Materials and Methods}

\subsection{Study Participants}

The study included patients older than 40 years with POAG or ocular hypertension (OHT) attending the Glaucoma Clinic at the Department of Ophthalmology of the University Medical Centre Ljubljana, Slovenia, from October 2018 until May 2020. The study was approved by the Slovenian Medical Ethics Committee (KME 30/05/11). All subjects signed informed consent in accordance with the Declaration of Helsinki.

Ocular hypertension was defined according to the Guidelines and Terminology of the European Glaucoma Society as IOP higher than $21 \mathrm{mmHg}$ without changes at the optic nerve head and visual field defects [46]. POAG was defined as untreated IOP higher than $21 \mathrm{mmHg}$, characteristic glaucoma changes at the optic nerve head and/or corresponding 
visual field defects. Patients with primary juvenile open-angle glaucoma, secondary causes of OHT/glaucoma and non-glaucomatous optic neuropathy and patients after prior intraocular surgery (except non-complicated cataract surgery with more than 6 months after surgery) were excluded. Patients were treated according to the recommendations of the European Glaucoma Society guidelines [47]. Lowering of the IOP was achieved by topical medications, laser trabeculoplasty or surgery, depending on the severity of glaucoma, life expectancy, status of the fellow eye and the rate of progression.

Data on the course of disease and treatment were obtained from medical records. In all patients, the following data were recorded: sex, age, smoking status, a family history of glaucoma, IOP, central corneal thickness (CCT), visual field parameters (with best-corrected visual acuity), vertical cup-disc (C/D) ratio, current ocular diagnosis and systemic diseases. The presence of diabetes, arterial hypertension $(\mathrm{AH})$, hyperlipidaemia and heart disease was also recorded.

The glaucoma phenotype was assessed with the severity of disease, C/D ratio, IOP and CCT. The severity of glaucoma was based on the visual field index mean defect (MD) of the Octopus standard automated perimetry ( $G$ program, dynamic strategy) and classified into early (MD $<6 \mathrm{~dB}$ ), moderate (MD 6-12 dB) and advanced (MD $>12 \mathrm{~dB}$ ) disease. The IOP was measured by Goldman applanation tonometry. The mean IOP based on all measurements during follow-up was calculated for each eye. CCT values were measured with a manual ultrasound pachymeter (Pachmate-DGH, Tehnology Inc., Exton, PA, USA).

The control group included 339 healthy, unrelated Slovenian blood donors without any systemic disease. For the control group, data about age and sex were available.

\subsection{DNA Isolation and Genotyping}

Nine candidate genes were selected based on their direct involvement in oxidative stress pathways and signalling cascades of inflammation. Only functional polymorphisms with a minor allele frequency above $5 \%$ were included in the study: non-synonymous SNPs in the coding region previously associated with enzyme activity or SNPs in the $5^{\prime}$ untranslated region previously associated with altered gene or protein expression levels were SOD2 rs4880, CAT rs1001179, GPX1 rs1050450, GSTP1 rs1695, IL1B rs1143623, IL1B rs16944, IL6 rs1800795 and TNF rs1800629 (Supplementary Materials Table S1) [48]. We also determined the presence of homozygous GSTM1 and GSTT1 gene deletion.

Genomic DNA was isolated from peripheral blood samples using the E.Z.N.A. ${ }^{\circledR}$ SQ II Blood DNA Kit (Omega Bio-tek, Inc., Norcross, GA, USA) following the manufacturer's instructions. GSTM1 and GSTT1 were genotyped using multiplex PCR that enabled the detection of homozygous gene deletion. In brief, both genes were simultaneously amplified in a single-step PCR together with the $\beta$-globin gene $(H B B)$ as the internal positive control, as previously described [49] (Table S2, Figure S1). Genotyping of 8 SNPs was performed with competitive allele-specific PCR (KASP), using the KASP Master mix and custom KASP genotyping assays (all from LGC, Middlesex, UK) according to the manufacturer's instructions (KBiosciences, Herts, UK and LGC Genomics, UK) (Table S2, Figure S2). Ten percent of the samples were genotyped in duplicate as quality control, and all the results were concordant.

\subsection{Statistical Analysis}

Continuous and categorical variables were described with the median and interquartile range (25-75\%) or frequencies, respectively. Fisher's exact test was used to compare the distribution of categorical variables, while nonparametric Mann-Whitney or KruskalWallis tests were used to compare the distribution of continuous variables. Deviation from the Hardy-Weinberg equilibrium (HWE) was evaluated using the standard chi-square test. Both dominant and additive genetic models were used in the analysis. The association of polymorphisms with glaucoma risk was evaluated using logistic regression to calculate non-adjusted and adjusted odds ratios (ORs) and 95\% confidence intervals (CIs). For the IOP, CCT and C/D ratio, data from the most affected eye were used in the analysis. If a 
measurement was available for only one eye, the measurement of that eye was used in the analysis.

All statistical tests were two sided. As 11 SNPs were investigated, the Bonferroni correction was used to account for multiple comparisons: $p$-values below 0.005 were considered statistically significant, while $p$-values between 0.005 and 0.050 were considered nominally significant. All statistical tests were two sided. In risk analysis, for a polymorphism with a minor allele frequency of $0.10,0.30$ and 0.50 , this study had $80 \%$ power to detect ORs of 1.9, 1.6 and 1.54 or more, respectively. Power calculation was conducted using PS Power and Sample Size Calculation version 3.0 [50]. The statistical analyses were carried out using IBM SPSS Statistics version 21.0 (IBM Corporation, Armonk, NY, USA).

\section{Results}

A total of 307 patients, 235 with POAG and 72 with OHT, participated in this study. The median age of patients was 70 (interquartile range 64-78) years. Among the patients, $139(45.3 \%)$ were male and $168(54.7 \%)$ were female. The control group consisted of 339 healthy blood donors with the median age of 49 (interquartile range 45-55) years. Among the controls, $251(74.0 \%)$ were male and $88(26.0 \%)$ were female. Patients with POAG or OHT were significantly older than controls $(p<0.001)$. There were more females among patients with POAG or OHT compared to controls $(p<0.001)$. In total, $31.9 \%$ of patients reported a family history of glaucoma. Clinical characteristics of all patients, their smoking history and accompanying systemic diseases are presented separately for the OHT and POAG groups in Table 1.

Table 1. Clinical characteristics of patients.

\begin{tabular}{|c|c|c|c|c|c|}
\hline Characteristic & & $\begin{array}{c}\text { Cases } \\
(n=307)\end{array}$ & $\begin{array}{c}\text { OHT } \\
(n=72)\end{array}$ & $\begin{array}{l}\text { POAG } \\
(n=235)\end{array}$ & $p$-Value \\
\hline \multirow[t]{2}{*}{ Sex } & Male, $n(\%)$ & $139(45.3)$ & $34(47.2)$ & $105(44.7)$ & 0.787 \\
\hline & Female, $n(\%)$ & $168(54.7)$ & $38(52.8)$ & $130(55.3)$ & \\
\hline Age (years) & Median (25-75\%) & $70(64-78)$ & $64(54-69)$ & $72(66-79)$ & $<0.001$ \\
\hline \multirow[t]{2}{*}{$\mathrm{IOP}(\mathrm{mmHg})$} & Right eye, (median (25-75\%) & $19.71(16.8-23)$ & $22.6(20.1-24.2)$ & $18.59(16.3-22.0)$ & $<0.001$ \\
\hline & Left eye, (median (25-75\%) & $19.78(16.7-23)$ & $22.5(19.7-24.2)$ & $18.48(16.3-22.0)$ & $<0.001$ \\
\hline \multirow[t]{2}{*}{$\mathrm{CCT}(\mu \mathrm{m})$} & Right eye, (median (25-75\%) & $547(521-574)$ & $565.5(533-595)$ & $541(518-570)$ & $<0.001$ \\
\hline & Left eye, (median (25-75\%) & $548.5(522.5-577.0)\{1\}$ & $570.5(532.3-602.5)$ & $543(519.5-571.0)$ & $<0.001$ \\
\hline \multirow[t]{2}{*}{$\mathrm{C} / \mathrm{D}$ ratio } & Right eye, (median (25-75\%) & $0.8(0.5-0.9)$ & $0.35(0.3-0.5)$ & $0.9(0.7-1.0)$ & $<0.001$ \\
\hline & Left eye, (median (25-75\%) & $0.8(0.5-1)$ & $0.3(0.3-0.5)$ & $0.9(0.7-1.0)$ & $<0.001$ \\
\hline \multirow{2}{*}{$\begin{array}{l}\text { Family history } \\
\text { of glaucoma }\end{array}$} & No, $n(\%)$ & $209(68.1)$ & $51(70.8)$ & $158(67.2)$ & 0.665 \\
\hline & Yes, $n(\%)$ & $98(31.9)$ & $21(29.2)$ & $77(32.8)$ & \\
\hline \multirow{2}{*}{$\begin{array}{c}\text { Arterial } \\
\text { hypertension }\{1\}\end{array}$} & No, $n(\%)$ & $140(45.8)$ & $38(52.8 \%)$ & $102(43.6)$ & 0.179 \\
\hline & Yes, $n(\%)$ & $166(54.2)$ & $34(47.2 \%)$ & $132(56.4)$ & \\
\hline \multirow[t]{2}{*}{ Diabetes } & No, $n(\%)$ & $280(91.2)$ & $64(88.9 \%)$ & $216(91.9)$ & 0.476 \\
\hline & Yes, $n(\%)$ & $27(8.8)$ & $8(11.1 \%)$ & $19(8.1)$ & \\
\hline \multirow[t]{2}{*}{ Hyperlipidaemia } & No, $n(\%)$ & $209(68.1)$ & $53(73.6 \%)$ & $156(66.4)$ & 0.312 \\
\hline & Yes, $n(\%)$ & 98 (31.9) & $19(26.4 \%)$ & $79(3.6)$ & \\
\hline \multirow[t]{2}{*}{ Heart disease $\{1\}$} & No, $n(\%)$ & $240(78.4)$ & $63(87.5 \%)$ & 177 (75.6) & 0.034 \\
\hline & Yes, $n(\%)$ & $66(21.6)$ & $9(12.5 \%)$ & $57(24.4)$ & \\
\hline \multirow[t]{3}{*}{ Smoking } & No, $n(\%)$ & $204(66.4)$ & $40(55.6 \%)$ & $164(69.8)$ & 0.045 \\
\hline & Currently, $n(\%)$ & $26(8.5)$ & $6(8.3)$ & $20(8.5)$ & \\
\hline & Former, $n(\%)$ & $77(25.1)$ & $26(36.1)$ & $51(21.7)$ & \\
\hline
\end{tabular}

\{\}-missing data; OHT—ocular hypertension; POAG—primary open-angle glaucoma; IOP—intraocular pressure; CCT-central corneal thickness; C/D ratio-vertical cup-disc ratio.

Genotypes' distributions for the investigated SNPs in both patient and control groups are presented in Table 2. When susceptibility analysis was performed, GSTM1 gene deletion was nominally significantly associated with the risk for POAG or OHT. The car- 
riers of GSTM1 gene deletion had lower odds for developing POAG or OHT (OR = 0.50; $95 \% \mathrm{CI}=0.30-0.83 ; p=0.007$ ).

Table 2. Genotype frequencies of the control $(n=339)$ and patient $(n=307)$ groups with the risk of glaucoma or OHT.

\begin{tabular}{|c|c|c|c|c|c|}
\hline SNP & Genotype & $\begin{array}{c}\text { Controls } \\
n(\%)\end{array}$ & $\begin{array}{l}\text { Cases } \\
n(\%)\end{array}$ & OR $(95 \%$ CI $)$ adj. & $p_{\text {adj-Value }}$ \\
\hline \multirow[t]{4}{*}{ SOD2 rs4880 } & $\mathrm{CC}$ & $92(27.1)$ & $78(25.4)$ & Reference & \\
\hline & $\mathrm{CT}$ & $160(47.2)$ & $136(44.3)$ & $1.11(0.60-2.06)$ & 0.745 \\
\hline & TT & $87(25.7)$ & $93(30.3)$ & $1.15(0.58-2.26)$ & 0.690 \\
\hline & $\mathrm{CT}+\mathrm{TT}$ & $247(72.9)$ & $229(74.6)$ & $1.12(0.63-2.00)$ & 0.691 \\
\hline \multirow[t]{4}{*}{ CAT rs1001179 } & $\mathrm{CC}$ & $193(57.1)\{1\}$ & $184(59.9)$ & Reference & \\
\hline & $\mathrm{CT}$ & $122(36.1)$ & $105(34.2)$ & $0.95(0.56-1.60)$ & 0.840 \\
\hline & TT & $23(6.8)$ & $18(5.9)$ & $1.31(0.42-4.08)$ & 0.642 \\
\hline & $\mathrm{CT}+\mathrm{TT}$ & $145(42.9)$ & $123(40.1)$ & $0.99(0.59-1.63)$ & 0.954 \\
\hline \multirow[t]{4}{*}{ GPX1 rs1050450 } & $\mathrm{CC}$ & $170(50.1)$ & $157(51.1)$ & Reference & \\
\hline & $\mathrm{CT}$ & $130(38.3)$ & $125(40.7)$ & $0.86(0.51-1.47)$ & 0.592 \\
\hline & TT & 39 (11.5) & $25(8.1)$ & $0.49(0.19-1.28)$ & 0.146 \\
\hline & $\mathrm{CT}+\mathrm{TT}$ & $169(49.9)$ & $150(48.9)$ & $0.78(0.47-1.30)$ & 0.345 \\
\hline \multirow[t]{4}{*}{ GSTP1 rs1695 } & AA & $150(44.2)$ & $141(45.9)$ & Reference & \\
\hline & AG & $152(44.8)$ & $128(41.7)$ & $0.98(0.58-1.67)$ & 0.940 \\
\hline & GG & 37 (10.9) & $38(12.4)$ & $1.84(0.79-4.28)$ & 0.157 \\
\hline & $\mathrm{AG}+\mathrm{GG}$ & $189(55.8)$ & $166(54.1)$ & $1.11(0.67-1.83)$ & 0.686 \\
\hline \multirow[t]{2}{*}{ GSTP1 rs1138272 } & $\mathrm{CC}$ & $274(80.8)$ & $254(82.7)$ & Reference & \\
\hline & $\mathrm{CT}+\mathrm{TT}$ & $65(19.2)$ & 53 (17.3) & $1.25(0.65-2.41)$ & 0.513 \\
\hline \multirow[t]{2}{*}{$\begin{array}{l}\text { GSTM1 gene } \\
\text { deletion }\end{array}$} & No deletion & $136(40.1)$ & $150(48.9)$ & Reference & \\
\hline & Deletion & $203(59.9)$ & 157 (51.1) & $0.50(0.30-0.83)$ & 0.007 \\
\hline \multirow[t]{2}{*}{$\begin{array}{l}\text { GSTT1 gene } \\
\text { deletion }\end{array}$} & No deletion & $288(85)$ & $254(82.7)$ & Reference & \\
\hline & Deletion & $51(15)$ & $53(17.3)$ & $0.58(0.30-1.12)$ & 0.103 \\
\hline \multirow[t]{4}{*}{ IL1B rs1143623 } & GG & $174(51.3)$ & $145(47.2)$ & Reference & \\
\hline & GC & $136(40.1)$ & $135(44)$ & $1.25(0.74-2.11)$ & 0.408 \\
\hline & $\mathrm{CC}$ & $29(8.6)$ & $27(8.8)$ & $1.50(0.61-3.69)$ & 0.378 \\
\hline & $\mathrm{GC}+\mathrm{CC}$ & $165(48.7)$ & $162(52.8)$ & $1.29(0.78-2.12)$ & 0.323 \\
\hline \multirow[t]{4}{*}{ IL1B rs16944 } & $\mathrm{TT}$ & $44(13)$ & $44(14.3)$ & Reference & \\
\hline & $\mathrm{TC}$ & $145(42.8)$ & $143(46.6)$ & $0.69(0.32-1.47)$ & 0.332 \\
\hline & $\mathrm{CC}$ & $150(44.2)$ & $120(39.1)$ & $0.62(0.28-1.35)$ & 0.227 \\
\hline & $\mathrm{TC}+\mathrm{CC}$ & $295(87.0)$ & $263(85.7)$ & $0.65(0.32-1.34)$ & 0.248 \\
\hline \multirow[t]{4}{*}{ IL6 rs1800795 } & GG & $120(35.4)$ & $111(36.2)$ & Reference & \\
\hline & GC & $151(44.5)$ & $154(50.2)$ & $0.71(0.41-1.24)$ & 0.226 \\
\hline & $\mathrm{CC}$ & $68(20.1)$ & $42(13.7)$ & $0.67(0.32-1.39)$ & 0.277 \\
\hline & $\mathrm{GC}+\mathrm{CC}$ & $219(64.6)$ & $196(63.8)$ & $0.70(0.41-1.17)$ & 0.174 \\
\hline \multirow[t]{2}{*}{ TNF rs1800629 } & GG & $228(67.3)$ & $234(76.2)$ & Reference & \\
\hline & $\mathrm{GA}+\mathrm{AA}$ & $111(32.7)$ & $73(23.8)$ & $0.66(0.38-1.17)$ & 0.156 \\
\hline
\end{tabular}


Next, susceptibility analysis was performed separately for patients with POAG $(n=235)$ and OHT $(n=72)$, as shown in Table 3. The carriers of GSTM1 gene deletion had lower odds for developing OHT (OR $=0.43 ; 95 \% \mathrm{CI}=0.22-0.81 ; p=0.009)$ but not for developing POAG $(\mathrm{OR}=0.61 ; 95 \% \mathrm{CI}=0.31-1.18 ; p=0.141)$.

Table 3. Genotype frequencies of selected polymorphisms and their association with the risk of OHT $(n=72)$ and POAG $(n=235)$.

\begin{tabular}{|c|c|c|c|c|c|c|c|}
\hline \multirow[b]{2}{*}{ SNP } & \multirow[b]{2}{*}{ Genotype } & \multicolumn{3}{|c|}{ OHT } & \multicolumn{3}{|c|}{ POAG } \\
\hline & & $n(\%)$ & OR (95\% CI) adj. & $p_{\text {adj }}$-Value & $n(\%)$ & OR (95\% CI) adj. & $p_{\text {adj }}$-Value \\
\hline \multirow[t]{4}{*}{ SOD2 rs4880 } & $\mathrm{CC}$ & $15(20.8)$ & Reference & & $63(26.8)$ & Reference & \\
\hline & $\mathrm{CT}$ & $39(54.2)$ & $1.37(0.63-3.00)$ & 0.426 & 97 (41.3) & $0.90(0.41-2.02)$ & 0.806 \\
\hline & TT & $18(25)$ & $1.12(0.46-2.71)$ & 0.798 & $75(31.9)$ & $1.23(0.51-2.93)$ & 0.643 \\
\hline & $\mathrm{CT}+\mathrm{TT}$ & $57(79.2)$ & $1.28(0.61-2.66)$ & 0.515 & $172(73.2)$ & $1.02(0.49-2.14)$ & 0.953 \\
\hline \multirow[t]{4}{*}{ CAT rs1001179 } & $\mathrm{CC}$ & $49(68.1)$ & Reference & & $135(57.4)$ & Reference & \\
\hline & $\mathrm{CT}$ & $20(27.8)$ & $0.66(0.33-1.30)$ & 0.230 & $85(36.2)$ & $1.36(0.68-2.69)$ & 0.381 \\
\hline & $\mathrm{TT}$ & $3(4.2)$ & $0.40(0.07-2.19)$ & 0.290 & $15(6.4)$ & $3.79(0.92-15.55)$ & 0.064 \\
\hline & $\mathrm{CT}+\mathrm{TT}$ & $23(31.9)$ & $0.62(0.32-1.20)$ & 0.157 & $100(42.6)$ & $1.54(0.80-2.96)$ & 0.201 \\
\hline \multirow[t]{4}{*}{ GPX1 rs1050450 } & $\mathrm{CC}$ & $45(62.5)$ & Reference & & $112(47.7)$ & Reference & \\
\hline & $\mathrm{CT}$ & $22(30.6)$ & $0.66(0.33-1.30)$ & 0.228 & $103(43.8)$ & $0.93(0.47-1.85)$ & 0.834 \\
\hline & TT & $5(6.9)$ & $0.36(0.10-1.26)$ & 0.111 & $20(8.5)$ & $0.70(0.20-2.44)$ & 0.581 \\
\hline & $\mathrm{CT}+\mathrm{TT}$ & $27(37.5)$ & $0.59(0.31-1.12)$ & 0.104 & $123(52.3)$ & $0.89(0.46-1.72)$ & 0.725 \\
\hline \multirow[t]{4}{*}{ GSTP1 rs1695 } & AA & $38(52.8)$ & Reference & & $103(43.8)$ & Reference & \\
\hline & AG & $26(36.1)$ & $0.92(0.47-1.79)$ & 0.800 & $102(43.4)$ & $0.92(0.46-1.84)$ & 0.819 \\
\hline & GG & $8(11.1)$ & $1.37(0.49-3.83)$ & 0.554 & $30(12.8)$ & $2.70(0.85-8.60)$ & 0.092 \\
\hline & $\mathrm{AG}+\mathrm{GG}$ & $34(47.2)$ & $0.99(0.53-1.86)$ & 0.987 & $132(56.2)$ & $1.11(0.58-2.13)$ & 0.747 \\
\hline \multirow[t]{2}{*}{$\begin{array}{c}\text { GSTP1 } \\
\text { rs1138272 }\end{array}$} & $\mathrm{CC}$ & $62(86.1)$ & Reference & & $192(81.7)$ & Reference & \\
\hline & $\mathrm{CT}+\mathrm{TT}$ & $10(13.9)$ & $1.09(0.47-2.55)$ & 0.837 & $43(18.3)$ & $1.44(0.60-3.47)$ & 0.410 \\
\hline \multirow[t]{2}{*}{$\begin{array}{c}\text { GSTM1 gene } \\
\text { deletion }\end{array}$} & No deletion & $36(50)$ & Reference & & $114(48.5)$ & Reference & \\
\hline & Deletion & $36(50)$ & $0.43(0.22-0.81)$ & 0.009 & $121(51.5)$ & $0.61(0.31-1.18)$ & 0.141 \\
\hline \multirow[t]{2}{*}{$\begin{array}{l}\text { GSTT1 gene } \\
\text { deletion }\end{array}$} & No deletion & $60(83.3)$ & Reference & & $194(82.6)$ & Reference & \\
\hline & Deletion & $12(16.7)$ & $0.61(0.26-1.44)$ & 0.258 & $41(17.4)$ & $0.50(0.22-1.16)$ & 0.107 \\
\hline \multirow[t]{4}{*}{ IL1B rs1143623 } & GG & $38(52.8)$ & Reference & & $107(45.5)$ & Reference & \\
\hline & GC & $32(44.4)$ & $1.16(0.61-2.19)$ & 0.657 & $103(43.8)$ & $1.12(0.56-2.24)$ & 0.745 \\
\hline & $\mathrm{CC}$ & $2(2.8)$ & $0.47(0.10-2.24)$ & 0.341 & 25 (10.6) & $2.87(0.95-8.65)$ & 0.061 \\
\hline & $\mathrm{GC}+\mathrm{CC}$ & $34(47.2)$ & $1.04(0.56-1.95)$ & 0.893 & $128(54.5)$ & $1.34(0.70-2.57)$ & 0.384 \\
\hline \multirow[t]{4}{*}{ IL1B rs16944 } & TT & $5(6.9)$ & Reference & & $39(16.6)$ & Reference & \\
\hline & $\mathrm{TC}$ & 34 (47.2) & $1.38(0.45-4.28)$ & 0.574 & $109(46.4)$ & $0.34(0.13-0.90)$ & 0.030 \\
\hline & $\mathrm{CC}$ & $33(45.8)$ & $1.44(0.46-4.47)$ & 0.533 & $87(37)$ & $0.32(0.12-0.86)$ & 0.024 \\
\hline & $\mathrm{TC}+\mathrm{CC}$ & $67(93.1)$ & $1.41(0.48-4.16)$ & 0.536 & $196(83.4)$ & $0.33(0.13-0.82)$ & 0.017 \\
\hline \multirow[t]{4}{*}{ IL6 rs1800795 } & GG & $25(34.7)$ & Reference & & $86(36.6)$ & Reference & \\
\hline & GC & $36(50)$ & $0.89(0.45-1.80)$ & 0.755 & $118(50.2)$ & $0.44(0.21-0.93)$ & 0.031 \\
\hline & $\mathrm{CC}$ & $11(15.3)$ & $0.77(0.30-1.96)$ & 0.577 & $31(13.2)$ & $0.49(0.19-1.29)$ & 0.149 \\
\hline & $\mathrm{GC}+\mathrm{CC}$ & 47 (65.3) & $0.86(0.44-1.66)$ & 0.654 & $149(63.4)$ & $0.46(0.23-0.91)$ & 0.025 \\
\hline \multirow[t]{2}{*}{ TNF rs1800629 } & GG & $56(77.8)$ & Reference & & $178(75.7)$ & Reference & \\
\hline & $\mathrm{GA}+\mathrm{AA}$ & $16(22.2)$ & $0.62(0.30-1.28)$ & 0.196 & $57(24.3)$ & $0.67(0.31-1.41)$ & 0.289 \\
\hline
\end{tabular}

adj—adjusted for age and sex; OHT—ocular hypertension; POAG—-primary open-angle glaucoma; SNP—single nucleotide polymorphism. Nominally significant values have been marked in bold. 
IL6 and IL1B polymorphisms showed nominally significant association with POAG but not with OHT. The carriers of the IL1B rs16944 polymorphism had lower odds for developing POAG in the dominant and additive genetic model ( $\mathrm{OR}=0.33 ; 95 \% \mathrm{CI}=0.13-0.82$; $p=0.017)$. IL6 rs 1800795 was associated with lower odds for developing POAG in the dominant genetic model $(\mathrm{OR}=0.46 ; 95 \% \mathrm{CI}=0.23-0.91 ; p=0.025)$ (Table 3$)$.

The distribution of genotype frequencies for the investigated polymorphisms was compared between groups of patients with OHT without visual field defects and patients with glaucoma of different severity (Table 4). The results showed statistically significant differences among groups only for the distribution of GPX1 rs1050450 genotypes in the dominant model. The carriers of at least one polymorphic GPX1 allele had less OHT and more POAG $(p=0.025$; Table 4$)$.

Table 4. Association of selected polymorphisms with OHT and POAG severity.

\begin{tabular}{|c|c|c|c|c|c|c|}
\hline SNP & Genotype & $\begin{array}{c}\text { OHT } \\
(n=72)\end{array}$ & $\begin{array}{c}\text { Early POAG } \\
(n=62)\end{array}$ & $\begin{array}{c}\text { Moderate POAG } \\
(n=54)\end{array}$ & $\begin{array}{c}\text { Severe } \\
\text { POAG }(n=119)\end{array}$ & $p$-Value * \\
\hline & & $n(\%)$ & $n(\%)$ & $n(\%)$ & $n(\%)$ & \\
\hline \multirow[t]{4}{*}{ SOD2 rs4880 } & $\mathrm{CC}$ & $15(19.2)$ & $15(19.2)$ & $15(19.2)$ & $33(42.3)$ & Padd $=0.681$ \\
\hline & $\mathrm{CT}$ & $39(28.7)$ & $27(19.9)$ & $22(16.2)$ & $48(35.3)$ & \\
\hline & $\mathrm{TT}$ & $18(19.4)$ & $20(21.5)$ & $17(18.3)$ & $38(40.9)$ & \\
\hline & $\mathrm{CT}+\mathrm{TT}$ & $57(24.9)$ & $47(20.5)$ & $39(17)$ & $86(37.6)$ & Pdom $=0.722$ \\
\hline \multirow[t]{4}{*}{ CAT rs1001179 } & $\mathrm{CC}$ & $49(26.6)$ & $31(16.8)$ & $35(19)$ & $69(37.5)$ & Padd $=0.149$ \\
\hline & $\mathrm{CT}$ & $20(19)$ & $23(21.9)$ & $18(17.1)$ & $44(41.9)$ & \\
\hline & TT & $3(16.7)$ & $8(44.4)$ & $1(5.6)$ & $6(33.3)$ & \\
\hline & $\mathrm{CT}+\mathrm{TT}$ & $23(18.7)$ & $31(25.2)$ & $19(15.4)$ & $50(40.7)$ & Pdom $=0.158$ \\
\hline \multirow[t]{4}{*}{ GPX1 rs1050450 } & $\mathrm{CC}$ & $45(28.7)$ & $33(21)$ & $19(12.1)$ & $60(38.2)$ & Padd $=0.134$ \\
\hline & $\mathrm{CT}$ & $22(17.6)$ & $24(19.2)$ & $29(23.2)$ & $50(40)$ & \\
\hline & TT & $5(20)$ & $5(20)$ & $6(24)$ & $9(36)$ & \\
\hline & $\mathrm{CT}+\mathrm{TT}$ & $27(18)$ & 29 (19.3) & $35(23.3)$ & $59(39.3)$ & Pdom $=0.025$ \\
\hline \multirow[t]{4}{*}{ GSTP1 rs1695 } & AA & $38(27)$ & $26(18.4)$ & $23(16.3)$ & $54(38.3)$ & Padd $=0.628$ \\
\hline & AG & $26(20.3)$ & $30(23.4)$ & $21(16.4)$ & $51(39.8)$ & \\
\hline & GG & $8(21.1)$ & $6(15.8)$ & $10(26.3)$ & $14(36.8)$ & \\
\hline & $\mathrm{AG}+\mathrm{GG}$ & $34(20.5)$ & $36(21.7)$ & $31(18.7)$ & 65 (39.2) & Pdom $=0.570$ \\
\hline \multirow[t]{2}{*}{$\begin{array}{c}\text { GSTP1 } \\
\text { rs1138272 }\end{array}$} & $\mathrm{CC}$ & $62(24.4)$ & 49 (19.3) & $41(16.1)$ & $102(40.2)$ & 0.299 \\
\hline & $\mathrm{CT}+\mathrm{TT}$ & $10(18.9)$ & $13(24.5)$ & $13(24.5)$ & $17(32.1)$ & \\
\hline \multirow[t]{2}{*}{$\begin{array}{c}\text { GSTM1 gene } \\
\text { deletion }\end{array}$} & No deletion & $36(24)$ & $23(15.3)$ & $30(20)$ & $61(40.7)$ & 0.195 \\
\hline & Deletion & $36(22.9)$ & $39(24.8)$ & 24 (15.3) & $58(36.9)$ & \\
\hline \multirow[t]{2}{*}{$\begin{array}{c}\text { GSTT1 gene } \\
\text { deletion }\end{array}$} & No deletion & $60(23.6)$ & $50(19.7)$ & $46(18.1)$ & $98(38.6)$ & 0.940 \\
\hline & Deletion & $12(22.6)$ & $12(22.6)$ & $8(15.1)$ & $21(39.6)$ & \\
\hline \multirow[t]{4}{*}{$I L 1 B$ rs1143623 } & GG & $38(26.2)$ & 27 (18.6) & $22(15.2)$ & $58(40)$ & Padd $=0.358$ \\
\hline & GC & $32(23.7)$ & $27(20)$ & $26(19.3)$ & $50(37)$ & \\
\hline & $\mathrm{CC}$ & $2(7.4)$ & $8(29.6)$ & $6(22.2)$ & $11(40.7)$ & \\
\hline & $\mathrm{GC}+\mathrm{CC}$ & $34(21)$ & $35(21.6)$ & $32(19.8)$ & $61(37.7)$ & Pdom $=0.526$ \\
\hline \multirow[t]{4}{*}{ IL1B rs16944 } & TT & $5(11.4)$ & $14(31.8)$ & $7(15.9)$ & $18(40.9)$ & Padd $=0.074$ \\
\hline & $\mathrm{TC}$ & $34(23.8)$ & $30(21)$ & $30(21)$ & $49(34.3)$ & \\
\hline & $\mathrm{CC}$ & 33 (27.5) & $18(15)$ & $17(14.2)$ & $52(43.3)$ & \\
\hline & $\mathrm{TC}+\mathrm{CC}$ & $67(25.5)$ & $48(18.3)$ & 47 (17.9) & $101(38.4)$ & Pdom $=0.079$ \\
\hline
\end{tabular}


Table 4. Cont.

\begin{tabular}{|c|c|c|c|c|c|c|}
\hline SNP & Genotype & $\begin{array}{c}\text { OHT } \\
(n=72)\end{array}$ & $\begin{array}{l}\text { Early POAG } \\
\quad(n=62)\end{array}$ & $\begin{array}{c}\text { Moderate POAG } \\
(n=54)\end{array}$ & $\begin{array}{c}\text { Severe } \\
\text { POAG }(n=119)\end{array}$ & $p$-Value * \\
\hline \multirow[t]{4}{*}{ IL6 rs1800795 } & GG & $25(22.5)$ & 24 (21.6) & $23(20.7)$ & $39(35.1)$ & Padd $=0.862$ \\
\hline & GC & $36(23.4)$ & $31(20.1)$ & $23(14.9)$ & $64(41.6)$ & \\
\hline & $\mathrm{CC}$ & $11(26.2)$ & 7 (16.7) & $8(19)$ & $16(38.1)$ & \\
\hline & $\mathrm{GC}+\mathrm{CC}$ & $47(24)$ & $38(19.4)$ & $31(15.8)$ & $80(40.8)$ & Pdom $=0.617$ \\
\hline \multirow[t]{2}{*}{ TNF rs1800629 } & GG & $56(23.9)$ & 46 (19.7) & 43 (18.4) & $89(38)$ & 0.873 \\
\hline & $\mathrm{GA}+\mathrm{AA}$ & $16(21.9)$ & $16(21.9)$ & $11(15.1)$ & $30(41.1)$ & \\
\hline
\end{tabular}

* Comparison of all 4 groups using Fisher's exact test; add-additive model; dom-dominant model; OHT-ocular hypertension; POAG - primary open-angle glaucoma; SNP—single nucleotide polymorphism. Statistically significant values have been marked in bold.

We also analysed the association of the investigated polymorphisms with the glaucoma phenotype, such as the IOP, CCT and C/D ratio (Table 5). The results showed a statistically significant association of GPX1 rs1050450 with the IOP and C/D ratio in the dominant model. The carriers of at least one polymorphic GPX1 allele had a lower IOP $(p=0.019)$ and a slightly increased $C / D$ ratio $(p=0.035)$. In addition, a statistically significant association of TNF rs1800629 with the CCT was found in the dominant model. The carriers of at least one polymorphic TNF rs1800629 allele had a larger CCT $(p=0.001)$.

Table 5. Association of investigated polymorphisms with the IOP, CCT and C/D ratio.

\begin{tabular}{|c|c|c|c|c|c|c|c|}
\hline SNP & Genotype & $\begin{array}{l}\text { IOP Max } \\
\text { Median } \\
(25-75 \%)\end{array}$ & $p$-Value & $\begin{array}{l}\text { CCT Min. } \\
\text { Median } \\
(25-75 \%)\end{array}$ & $p$-Value & $\begin{array}{l}\text { C/D Max. } \\
\text { Median } \\
(25-75 \%)\end{array}$ & $p$-Value \\
\hline \multirow[t]{4}{*}{ SOD2 rs4880 } & $\mathrm{CC}$ & $21(17.5-26)$ & Padd $=0.301$ & $\begin{array}{c}539 \\
(520.75-571)\end{array}$ & Padd $=0.422$ & $0.85(0.6-1)$ & Padd $=0.246$ \\
\hline & $\mathrm{CT}$ & $20.8(17.5-23.3)$ & & $548(520-577)$ & & $0.8(0.5-1)$ & \\
\hline & TT & $20.2(17.3-23)$ & & $541(514.5-573)$ & & $0.9(0.7-1)$ & \\
\hline & $\mathrm{CT}+\mathrm{TT}$ & $20.6(17.5-23)$ & Pdom $=0.139$ & $544(518.5-573)$ & Pdom $=0.590$ & $0.9(0.6-1)$ & Pdom $=0.985$ \\
\hline \multirow[t]{4}{*}{ CAT rs1001179 } & $\mathrm{CC}$ & $20.8(17.5-23.7)$ & Padd = 0.574 & $\begin{array}{c}543 \\
(518.25-573.75)\end{array}$ & Padd $=0.331$ & $0.85(0.6-1)$ & Padd $=0.517$ \\
\hline & $\mathrm{CT}$ & $20.6(17.4-23.7)$ & & $\begin{array}{c}541 \\
(519.5-571.5)\end{array}$ & & $0.9(0.6-1)$ & \\
\hline & $\mathrm{TT}$ & $22.5(17.5-26)$ & & $\begin{array}{c}557 \\
(532.5-584.5)\end{array}$ & & $0.8(0.6-0.9)$ & \\
\hline & $\mathrm{CT}+\mathrm{TT}$ & $20.8(17.5-24)$ & Pdom $=0.759$ & $544(520-573)$ & Pdom $=0.994$ & $0.9(0.6-1)$ & Pdom $=0.690$ \\
\hline \multirow[t]{4}{*}{$\begin{array}{c}G P X 1 \\
\text { rs1050450 }\end{array}$} & $\mathrm{CC}$ & $21.2(17.9-24)$ & Padd $=0.061$ & $551(520.5-575)$ & Padd $=0.245$ & $0.8(0.5-1)$ & Padd $=0.105$ \\
\hline & CT & $19.4(17.2-23)$ & & $538(519-571)$ & & $0.9(0.7-1)$ & \\
\hline & TT & $19(17.2-24)$ & & $540(501.5-572)$ & & $0.9(0.6-1)$ & \\
\hline & $\mathrm{CT}+\mathrm{TT}$ & $19.3(17.2-23.2)$ & Pdom $=0.019$ & $538.5(518-571)$ & Pdom $=0.094$ & $0.9(0.675-1)$ & Pdom $=0.035$ \\
\hline \multirow[t]{4}{*}{ GSTP1 rs1695 } & AA & $20.5(17.6-23.5)$ & Padd $=0.991$ & $546(519.5-575)$ & Padd $=0.154$ & $0.8(0.55-1)$ & Padd $=0.572$ \\
\hline & $\mathrm{AG}$ & $21(17.2-24)$ & & $\begin{array}{c}537.5 \\
(515.5-570)\end{array}$ & & $0.9(0.6-1)$ & \\
\hline & GG & $20.1(17.7-24.1)$ & & $\begin{array}{c}547.5 \\
(531-574.75)\end{array}$ & & $0.9(0.7-1)$ & \\
\hline & $\mathrm{AG}+\mathrm{GG}$ & $20.9(17.4-24)$ & Pdom $=0.899$ & $\begin{array}{c}540.5 \\
(520-572.25)\end{array}$ & Pdom $=0.598$ & $0.9(0.6-1)$ & Pdom $=0.357$ \\
\hline \multirow[t]{2}{*}{$\begin{array}{c}\text { GSTP1 } \\
\text { rs1138272 }\end{array}$} & $\mathrm{CC}$ & $20.6(17.5-24)$ & 0.924 & $544(520-573)$ & 0.965 & $0.9(0.6-1)$ & 0.846 \\
\hline & $\mathrm{CT}+\mathrm{TT}$ & $21(17.4-23.7)$ & & $\begin{array}{c}540 \\
(519.5-575.5)\end{array}$ & & $0.9(0.6-1)$ & \\
\hline \multirow[t]{2}{*}{$\begin{array}{l}\text { GSTM1 gene } \\
\text { deletion }\end{array}$} & No deletion & $21(17.2-24)$ & 0.967 & $544(515-576)$ & 0.894 & $0.9(0.6-1)$ & 0.823 \\
\hline & Deletion & $20.6(17.6-23.5)$ & & $541(520-572.5)$ & & $0.8(0.6-1)$ & \\
\hline
\end{tabular}


Table 5. Cont.

\begin{tabular}{|c|c|c|c|c|c|c|c|}
\hline SNP & Genotype & $\begin{array}{l}\text { IOP Max } \\
\text { Median } \\
(25-75 \%)\end{array}$ & $p$-Value & $\begin{array}{l}\text { CCT Min. } \\
\text { Median } \\
(25-75 \%)\end{array}$ & $p$-Value & $\begin{array}{l}\text { C/D Max. } \\
\text { Median } \\
(25-75 \%)\end{array}$ & $p$-Value \\
\hline \multirow[t]{2}{*}{$\begin{array}{l}\text { GSTT1 gene } \\
\text { deletion }\end{array}$} & No deletion & $20.7(17.4-23.9)$ & 0.354 & $541.5(518-573)$ & 0.199 & $0.9(0.6-1)$ & 0.994 \\
\hline & Deletion & $21(18-24)$ & & $544(529.5-577)$ & & $0.9(0.6-1)$ & \\
\hline \multirow[t]{4}{*}{ IL1B rs1143623 } & GG & $21(17.6-24)$ & Padd $=0.268$ & $539(518-575)$ & Padd $=0.382$ & $0.9(0.6-1)$ & Padd $=0.843$ \\
\hline & GC & $20.2(17.2-23)$ & & $544(520-571)$ & & $0.8(0.6-1)$ & \\
\hline & $\mathrm{CC}$ & $21(17.6-24)$ & & $557(530-573)$ & & $0.9(0.6-1)$ & \\
\hline & $\mathrm{GC}+\mathrm{CC}$ & $20.3(17.3-23)$ & Pdom $=0.128$ & $547(521-571.5)$ & Pdom $=0.383$ & $0.85(0.6-1)$ & Pdom $=0.970$ \\
\hline \multirow[t]{4}{*}{$I L 1 B$ rs16944 } & TT & $20.4(17.7-23.1)$ & Padd $=0.411$ & $\begin{array}{c}551 \\
(529.25-570)\end{array}$ & Padd $=0.617$ & $0.9(0.7-1)$ & Padd $=0.541$ \\
\hline & $\mathrm{TC}$ & $20.4(17.5-23)$ & & $544(520-574)$ & & $0.8(0.6-1)$ & \\
\hline & $\mathrm{CC}$ & $21(17.2-25)$ & & $\begin{array}{c}538.5 \\
(518-573.75) \\
\end{array}$ & & $0.9(0.5-1)$ & \\
\hline & $\mathrm{TC}+\mathrm{CC}$ & $20.8(17.4-24)$ & Pdom $=0.989$ & $541(519-574)$ & Pdom $=0.508$ & $0.9(0.6-1)$ & Pdom $=0.319$ \\
\hline \multirow[t]{4}{*}{ IL6 rs1800795 } & GG & $20(17.6-23.3)$ & Padd $=0.184$ & $546(520-578)$ & Padd $=0.354$ & $0.8(0.5-1)$ & Padd $=0.342$ \\
\hline & GC & $20.6(17.2-23.4)$ & & $\begin{array}{c}541.5 \\
(520.75-573)\end{array}$ & & $0.9(0.6-1)$ & \\
\hline & $\mathrm{CC}$ & $21.8(18.4-26)$ & & $\begin{array}{c}534 \\
(502.75-569.25)\end{array}$ & & $0.8(0.4-1)$ & \\
\hline & $\mathrm{GC}+\mathrm{CC}$ & $21(17.3-24)$ & Pdom $=0.491$ & $540.5(518-571)$ & Pdom $=0.390$ & $0.9(0.6-1)$ & Pdom $=0.451$ \\
\hline \multirow[t]{2}{*}{ TNF rs1800629 } & GG & $20.5(17.2-23.2)$ & 0.166 & $\begin{array}{c}540 \\
(517.75-570)\end{array}$ & 0.001 & $0.9(0.6-1)$ & 0.539 \\
\hline & $\mathrm{GA}+\mathrm{AA}$ & $21.7(17.7-24.1)$ & & $\begin{array}{c}564 \\
(529.5-589.5)\end{array}$ & & $0.9(0.6-1)$ & \\
\hline
\end{tabular}

add-additive model; dom-dominant model; IOP_-intraocular pressure; CCT-central corneal thickness; C/D ratio-vertical cup-disc ratio; SNP_single nucleotide polymorphism. Statistically significant values have been marked in bold.

We also analysed the association between patients' clinical characteristics and the glaucoma phenotype. Cases with $\mathrm{AH}$ had a significantly lower maximal median IOP compared to those without $\mathrm{AH}(p<0.001)$ and a greater C/D ratio $(p=0.008)$ (Table 6). Similarly, ischaemic heart disease was significantly associated with lower IOP $(p=0.002)$. However, patients with glaucoma were older compared to OHT subjects $(p<0.001)$ with a higher prevalence of heart disease $(p=0.034)$. Diabetes was significantly associated with a larger CCT $(p=0.030)$.

Table 6. Association of patients' clinical characteristics with the glaucoma phenotype.

\begin{tabular}{|c|c|c|c|c|c|c|c|}
\hline Characteristic & & $\begin{array}{l}\text { IOP Max. } \\
\text { Median } \\
(25-75 \%)\end{array}$ & $p$-Value & $\begin{array}{l}\text { CCT Min. } \\
\text { Median } \\
(25-75 \%)\end{array}$ & $p$-Value & $\begin{array}{c}\text { C/D Ratio Max. } \\
\text { Median } \\
(25-75 \%)\end{array}$ & $p$-Value \\
\hline \multirow[t]{2}{*}{ Smoking } & No, $n(\%)$ & $20.1(17.5-23.7)$ & 0.356 & $541(520-573)$ & 0.589 & $0.9(0.6-1)$ & 0.019 \\
\hline & Yes, $n(\%)$ & $21(17.4-24)$ & & $544(520-573)$ & & $0.8(0.5-1)$ & \\
\hline \multirow[t]{2}{*}{$\begin{array}{l}\text { Family } \\
\text { history }\end{array}$} & No, $n(\%)$ & $21(17.7-24)$ & 0.073 & $544(520-574)$ & 0.632 & $0.9(0.6-1)$ & 0.695 \\
\hline & Yes, $n(\%)$ & $20(17.1-23.2)$ & & $\begin{array}{c}540.5 \\
(518.8-570.5)\end{array}$ & & $0.9(0.6-1)$ & \\
\hline \multirow[t]{2}{*}{$\mathrm{AH}$} & No, $n(\%)$ & $22(18.6-24.2)$ & $<0.001$ & $\begin{array}{c}548.5 \\
(526.8-575.5)\end{array}$ & 0.050 & $0.8(0.5-1)$ & 0.008 \\
\hline & Yes, $n(\%)$ & $19.4(16.9-22.8)$ & & $538(512-570.3)$ & & $0.9(0.6-1)$ & \\
\hline \multirow[t]{2}{*}{ Diabetes } & No, $n(\%)$ & $20.6(17.2-23.7)$ & 0.071 & $541(518.3-573)$ & 0.030 & $0.9(0.6-1)$ & 0.543 \\
\hline & Yes, $n(\%)$ & $22(19.3-24)$ & & $558(540-580)$ & & $0.9(0.4-1)$ & \\
\hline \multirow[t]{2}{*}{ Hyperlipidaemia } & No, $n(\%)$ & $21(17.6-24)$ & 0.078 & $543(518.5-574)$ & 0.959 & $0.8(0.6-1)$ & 0.541 \\
\hline & Yes, $n(\%)$ & 19.7 (17.2-23.1) & & $543.5(520-570)$ & & $0.9(0.6-1)$ & \\
\hline
\end{tabular}


Table 6. Cont

\begin{tabular}{|c|c|c|c|c|c|c|c|}
\hline Characteristic & & $\begin{array}{l}\text { IOP Max. } \\
\text { Median } \\
(25-75 \%)\end{array}$ & $p$-Value & $\begin{array}{l}\text { CCT Min. } \\
\text { Median } \\
(25-75 \%)\end{array}$ & $p$-Value & $\begin{array}{c}\text { C/D Ratio Max. } \\
\text { Median } \\
(25-75 \%)\end{array}$ & $p$-Value \\
\hline \multirow[t]{2}{*}{ Heart disease } & No, $n(\%)$ & $21(17.7-24)$ & 0.002 & $544.5(520-575.5)$ & 0.173 & $0.8(0.6-1)$ & 0.485 \\
\hline & Yes, $n(\%)$ & $19(16.7-21.5)$ & & $536.5(515.8-562.3)$ & & $0.9(0.6-1)$ & \\
\hline
\end{tabular}

$\mathrm{AH}$-arterial hypertension; IOP—intraocular pressure; CCT—central corneal thickness; C/D ratio—vertical cup-disc ratio. Statistically significant values have been marked in bold.

In the multivariable analysis including GPX1 rs1050450, $\mathrm{AH}$ and diabetes, both GPX1 rs1050450 ( $p=0.014)$ and patients' clinical characteristics remained significantly associated with the IOP ( $p<0.001$ and $p=0.019$, respectively). In the multivariable analysis including GPX1 rs1050450, AH and smoking, only GPX1 rs1050450 remained significantly associated with the $\mathrm{C} / \mathrm{D}$ ratio $(p=0.027)$. In the multivariable model including TNF rs1800629 and diabetes, both variables were significantly associated with the CCT $(p<0.001$ and $p=0.015)$.

\section{Discussion}

In this study, we evaluated the associations of selected SNPs in antioxidative and inflammation pathways with the risk of glaucoma and the glaucoma phenotype. Our main finding was that in the antioxidative pathways, GSTM1 gene deletion may play a protective role in the development of OHT, while inflammatory pathway polymorphisms such as IL1B rs16944 and IL6 rs1800795 may play a protective role in the development of POAG. Interestingly, GPX1 rs1050450 polymorphism was mainly associated with the severity of POAG and with the phenotype such as the IOP and C/D ratio.

In our study, we observed that the carriers of GSTM1 gene deletion had lower odds for developing OHT, but we found no association between GSTT1 gene deletion and the risk of OHT or POAG. GSTs play an important role in cellular protection against oxidative stress. Homozygous GSTM1 and GSTT1 deletion results in the absence of the encoded enzymes and may thus impair detoxification and inactivation of reactive metabolites generated during oxidative stress. GSTM1 and GSTT1 gene deletion was extensively investigated with regard to glaucoma risk. Whereas some studies have found a significantly higher frequency of the GSTM1 null genotype in patients with POAG, especially in smokers compared to controls [51-53], others have reported increased risk with a GSTM1 positive phenotype, or in combination with a GSTT1 null genotype, $[54,55]$ or did not find any association with the risk of glaucoma [56-58]. Many factors might account for the differences in results between similar studies, such as differences in sample size, types of glaucoma and ethnic and geographical GSTM1 null and GSTT1 null distribution in populations. A recent meta-analysis suggested that there might be a significant association between GSTM1 polymorphisms and increased susceptibility to glaucoma [57].

In our study, GPX1 rs1050450 was not associated with the risk of glaucoma. We also found no association between the CAT rs1001179 promoter variant and the risk of glaucoma. CAT and GPX1 directly participate in the inactivation of hydrogen peroxide, while GPX1 also participates in the detoxification of reactive secondary metabolites of oxidative stress, such as various lipid hydroperoxides. The most common GPX1 rs1050450 polymorphism codes for Pro198Leu substitution and leads to decreased enzyme activity; therefore, the capacity for antioxidant defence may also be decreased [59]. Defence capacity against ROS may also be decreased in carriers of a common functional CAT rs1001179 polymorphism that influences transcription factor binding in the promoter region and is associated with decreased catalase levels [60]. Associations of GPX1 polymorphisms have only been studied in other neurodegenerative diseases such as Alzheimer's disease [61] and Parkinson's disease [48] but not in glaucoma. Likewise, CAT rs1001179 genotype frequencies did not differ significantly between cases and controls in previous studies [24,62]. Only the synonymous SNP CAT rs769217 was significantly associated with POAG in the Chinese population [62]. We also found no association between SOD rs4880 and glaucoma. Simi- 
larly, no difference in the allele and genotype frequency in SNPs rs4880 between POAG cases and controls was reported by Zhou et al. [63].

With regard to inflammatory pathway polymorphisms, we found a protective effect of IL1B rs16944 and IL6 rs1800795 on the development of POAG. Both polymorphisms are located in the promoter region and may lead to altered gene expression. In patients with POAG, significantly increased mRNA expression of the IL1B gene has been found in blood and significantly increased IL-1B protein expression in the aqueous humour compared to controls [64]. In an animal model of acute glaucoma, upregulation of IL$1 \mathrm{~B}$ caused an increase in retinal ganglion cell death [65]. Polymorphisms in the IL1B promoter region (rs16944 and rs1143634-not analysed in our study) have already been investigated for an association with POAG. Whereas positive associations were reported in Caucasian populations [66,67], associations with POAG were not observed in Asian populations [68-71]. Meta-analysis evaluating the role of these two SNPs of IL1B in the susceptibility to glaucoma did not find any association, but the conclusions should be interpreted with caution as only a small number of studies was included [72].

With regard to IL6, Zimmermann et al. suggested that the promoter IL6 rs1800795 polymorphism is unlikely to be a major risk factor for POAG [73]. In addition, a recent systematic review and meta-analysis found a statistically significant glaucoma risk associated only with rs1524107, but not with rs1800795, which was investigated in our study [42]. However, when patients with early to moderate glaucoma were compared to patients with advanced glaucoma, the IL6 rs 1800795 C allele as well as the GC genotype were protective against less severe forms of normal-tension glaucoma [69].

We are not aware of any studies investigating the association of SNPs involved in the inflammatory and oxidative stress pathways with the glaucoma phenotype, including the severity of glaucoma, IOP, C/D ratio and CCT. We found a statistically significant association of GPX1 rs1050450 with the severity of glaucoma. In the dominant model, the frequency of at least one polymorphic GPX allele increased with the severity of glaucoma (19.3\% in early, $23.3 \%$ in moderate and $39.3 \%$ in advanced glaucoma). The GPX1 rs1050450 CT and TT genotypes were reported to be associated with increased risk of damage caused by oxidative stress, such as in coronary heart disease and cancer [74,75]. The GPX1 rs1050450 CT and TT genotypes were associated with increased risk of POAG in the Polish population, but the link with glaucoma phenotypes has not been investigated [76]. Antioxidant enzymes, such as GPX, are an important defence system against oxidative stress, which may play a major pathophysiological role in glaucoma. An increase in oxidative stress markers in serum and aqueous humour with a decrease in serum antioxidant stress markers was present in glaucoma patients compared to controls. However, despite a decrease in serum GPX, there was an important increase in GPX in the aqueous humour [11]. This may indicate a protective response of the eye against oxidative stress and may wear off in the long term [19].

Glaucoma is a heritable disease, and siblings of POAG cases have a tenfold-increased risk of developing the disease [77]. The C/D ratio, IOP and CCT used clinically to predict POAG risk are heritable traits related to the disease and may be associated with genetic variability in inflammation and oxidative stress pathways. In our study, carriers of at least one polymorphic GPX1 rs1050450 allele had statistically lower IOP and a lower C/D ratio. Patients with advanced glaucoma require lower target IOP to prevent progression of disease than those with early glaucoma or ocular hypertension [78]. In our study, approximately $30 \%$ of all cases had advanced disease. Therefore, those with lower IOP had presumably advanced glaucoma, which might partly explain the link between GPX1 polymorphisms and lower IOP and severity of glaucoma. However, the association between this SNP and the $\mathrm{C} / \mathrm{D}$ ratio is not clear. We found no previous studies analysing the association of the GPX1 SNP with the glaucoma phenotype.

In the last decade, genome-wide association studies (GWAS) have identified over 50 C/D-ratio-associated loci, but only 9 of these have been associated with POAG [79,80]. Up till now, multiple IOP-associated loci were identified using large and multi-ethnic 
biobank-based cohorts. Among the significant results were also loci at genes previously associated with POAG but not previously known to influence IOP. This indicates that genetic variation at these genes mediates the increased POAG risk via raised IOP rather than via direct effect on retinal ganglion cells $[79,80]$. The identified loci explained $17 \%$ of the variance of IOP in the EPIC-Norfolk Eye Study [81].

The central corneal thickness has been associated with increased POAG development and progression $[82,83]$, but it is uncertain whether this relationship is caused by IOP measurement artefacts or whether the relationship is biologically causal [84]. In our study, the GG genotype of TNF rs1800629 was associated with a low CCT. The link between TNF polymorphisms and the CCT has not been investigated. However, recently, the results of GWAS for the CCT have suggested that the CCT may not be a heritable trait for POAG and that the CCT-glaucoma association observed in studies is due to IOP measurement artefacts rather than biological causality [80].

Multiple epidemiological studies have reported the role of hypertension as a risk factor for POAG $[85,86]$. Treatment of hypertensive patients with beta-blockers results in nocturnal hypotension, which is a potential risk factor for glaucomatous optic neuropathy $[87,88]$. The mechanisms by which hypertension induces optic nerve damage are still unclear. Whether or not an association exists between diabetes mellitus and glaucoma has been an issue of debate, but findings from several studies in recent years suggest that the risk of glaucoma among diabetic patients may be greater than once believed [89-93]. Patients with POAG may suffer from ischaemic heart disease more often than those without glaucoma [93]. We also analysed the association between clinical characteristics (AH, diabetes, ischaemic heart disease, family history of glaucoma) with the glaucoma phenotype (IOP, $\mathrm{C} / \mathrm{D}$ ratio, $\mathrm{CCT}$ ). In our study, patients with $\mathrm{AH}$ had significantly lower maximal median IOP and a greater $\mathrm{C} / \mathrm{D}$ ratio compared to those without $\mathrm{AH}$. A possible explanation is that among cases with $\mathrm{AH}$, there were older patients with advanced glaucoma, requiring lower target IOP compared to cases with OHT. Similarly, in our study, patients with ischaemic heart disease had significantly lower IOP. As patients with glaucoma were older compared to OHT subjects and with a higher prevalence of heart disease, they required lower target IOP to prevent progression, which could explain our results. Diabetes was significantly associated with a larger CCT in our study. Our observation is in line with the findings of a meta-analysis that suggested that diabetes and hyperglycaemia are associated with a thicker cornea [94]. We found no other associations between the glaucoma phenotype or clinical characteristics with the investigated SNPs.

One of the limitations of our study was that the number of control subjects and cases was small compared to larger studies investigating genetic factors. Furthermore, we investigated only a limited number of polymorphisms in the oxidative stress and inflammation pathways. The strength of our study was that we used a pathway-based approach and selected SNPs with a known functional effect that are common in the Caucasian population. Perhaps other polymorphisms of the genes involved in these pathways, not investigated in our study, may have had a potential impact. Another limitation was that only data on age and sex were available for the control group. However, all controls were healthy blood donors without any self-reported systemic disease. Furthermore, the chance of controls having undiagnosed glaucoma was small due to the low prevalence of the disease in this age group, estimated to be between $0.2 \%$ and $1.1 \%$ in a Caucasian population of the same age [95-97]. Another strength of our study was that all patients attended the same glaucoma unit with the same treatment approach and follow-up, unambiguous diagnostic criteria and classification of phenotype. Furthermore, all patients and controls originated from a genetically homogenous population, thus limiting possible bias due to the population structure $[98,99]$.

Although our findings should be interpreted with caution, there is further perspective to this research. Ganglion cell death in glaucoma is a complex process triggered by different molecular mechanisms, among which oxidative stress and activation of inflammation by retinal glial cells play an important role. Improving antioxidant defence and addressing 
inflammation pathways might stimulate cell survival and boost the cells' ability to withstand pathological insult. Several studies have shown the potential protective effect of antioxidants on retinal ganglion cells [100-103], while there is experimental evidence that modulation of inflammation reduces retinal ganglion cell death [102]. Therefore, improved knowledge of these pathways might help to establish predictive biomarkers to improve treatment strategies for glaucoma. Patients could be stratified into groups with detectable deficits in oxidative stress and/or inflammation pathways, so supplementary therapy could be more specific and treatment personalised. Our study investigated genes and SNPs with broad implications in glaucoma and other neurodegenerative diseases that share similar biomarkers $[104,105]$. This type of study on glaucoma and similar diseases may help to design inflammation and oxidative stress pathway gene panels that could be used in testing patients with different but related diseases in order to personalise treatment and potentially improve treatment outcomes.

In conclusion, we used a pathway-based approach to address the relationship between oxidative stress and inflammation polymorphisms, and POAG risk. We found some indications for a possible association of genetic variability in GSTM1 with OHT. While IL1B and IL6 may be associated with the risk of glaucoma, GPX and TNF may affect the glaucoma phenotype. However, the evidence presented here is limited and further association and functional studies are required.

Supplementary Materials: The following are available online at https:/ / www.mdpi.com/2077-038 3/10/5/1148/s1, Table S1: Characteristics of investigated polymorphisms, variant allele frequency and agreement with Hardy-Weinberg equilibrium in controls, Table S2: Primers used for multiplex PCR (a) and thermal cycling conditions used for genotyping for multiplex PCR (b) and KASP chemistry (c), Figure S1: Representative gel image of GSTT1 and GSTM1 genotyping analysis, Figure S2: Representative cluster image for IL6 rs1800795 analysis obtained after KASP competitive allele specific PCR. References [106-111] are cited in the supplementary materials.

Author Contributions: Conceptualisation, V.D. and B.C.; methodology, V.D. and B.C.; software, K.G.; validation, M.A.V., V.D., K.G., T.B. and B.C.; formal analysis, M.A.V., K.G. and T.B.; investigation, M.A.V., K.G., V.D. and B.C.; resources, V.D. and B.C.; data curation, M.A.V., K.G. and T.B.; writingoriginal draft preparation, M.A.V. and B.C.; writing-M.A.V., K.G., V.D. and B.C.; review and editing, V.D. and B.C.; visualisation, M.A.V. and K.G.; supervision, V.D. and B.C.; project administration, M.A.V., K.G., V.D. and T.B.; funding acquisition, none. All authors have read and agreed to the published version of the manuscript.

Funding: This research was funded by the Slovenian Research Agency (ARRS) (grant nos. P1-0170 and P3-0333).

Institutional Review Board Statement: The study was conducted according to the guidelines of the Declaration of Helsinki and approved by the Slovenian Medical Ethics Committee (KME 30/05/11).

Informed Consent Statement: Informed consent was obtained from all subjects involved in the study.

Data Availability Statement: The datasets used and/or analysed for the current study are available from the corresponding author on reasonable request.

Acknowledgments: We thank all the patients for their participation in the study. We would like to thank Saša Mohar, head nurse of the glaucoma unit, for contribution to patient enrolment. We would also like to thank Savica Soldat from the pharmacogenetics laboratory for help with the laboratory work.

Conflicts of Interest: The authors declare no conflict of interest.

\section{References}

1. Pinazo-Duran, M.D.; Zanon-Moreno, V.; Garcia-Medina, J.J.; Gallego-Pinazo, R. Evaluation of presumptive biomarkers of oxidative stress, immune response and apoptosis in primary open-angle glaucoma. Curr. Opin. Pharmacol. 2013, 13, 98-107. [CrossRef] [PubMed]

2. Gupta, N.; Ang, L.C.; Noel de Tilly, L.; Bidaisee, L.; Yucel, Y.H. Human glaucoma and neural degeneration in intracranial optic nerve, lateral geniculate nucleus, and visual cortex. Br. J. Ophthalmol. 2006, 90, 674-678. [CrossRef] 
3. Gupta, N.; Ly, T.; Zhang, Q.; Kaufman, P.L.; Weinreb, R.N.; Yucel, Y.H. Chronic ocular hypertension induces dendrite pathology in the lateral geniculate nucleus of the brain. Exp. Eye Res. 2007, 84, 176-184. [CrossRef] [PubMed]

4. Gupta, N.; Fong, J.; Ang, L.C.; Yucel, Y.H. Retinal tau pathology in human glaucomas. Can. J. Ophthalmol. 2008, 43, 53-60. [CrossRef]

5. Gallego, B.I.; Salazar, J.J.; de Hoz, R.; Rojas, B.; Ramirez, A.I.; Salinas-Navarro, M.; Ortin-Martinez, A.; Valiente-Soriano, F.J.; Aviles-Trigueros, M.; Villegas-Perez, M.P.; et al. IOP induces upregulation of GFAP and MHC-II and microglia reactivity in mice retina contralateral to experimental glaucoma. J. Neuroinflamm. 2012, 9, 92. [CrossRef]

6. De Hoz, R.; Gallego, B.I.; Ramirez, A.I.; Rojas, B.; Salazar, J.J.; Valiente-Soriano, F.J.; Aviles-Trigueros, M.; Villegas-Perez, M.P.; Vidal-Sanz, M.; Trivino, A.; et al. Rod-like microglia are restricted to eyes with laser-induced ocular hypertension but absent from the microglial changes in the contralateral untreated eye. PLoS ONE 2013, 8, e83733. [CrossRef]

7. Rojas, B.; Gallego, B.I.; Ramirez, A.I.; Salazar, J.J.; de Hoz, R.; Valiente-Soriano, F.J.; Aviles-Trigueros, M.; Villegas-Perez, M.P.; Vidal-Sanz, M.; Trivino, A.; et al. Microglia in mouse retina contralateral to experimental glaucoma exhibit multiple signs of activation in all retinal layers. J. Neuroinflamm. 2014, 11, 133. [CrossRef] [PubMed]

8. Tezel, G. Oxidative stress in glaucomatous neurodegeneration: Mechanisms and consequences. Prog. Retin. Eye Res. 2006, 25, 490-513. [CrossRef] [PubMed]

9. Izzotti, A.; Bagnis, A.; Sacca, S.C. The role of oxidative stress in glaucoma. Mutat. Res. 2006, 612, 105-114. [CrossRef] [PubMed]

10. Ghanem, A.A.; Arafa, L.F.; El-Baz, A. Oxidative stress markers in patients with primary open-angle glaucoma. Curr. Eye Res. 2010, 35, 295-301. [CrossRef]

11. Benoist d'Azy, C.; Pereira, B.; Chiambaretta, F.; Dutheil, F. Oxidative and Anti-Oxidative Stress Markers in Chronic Glaucoma: A Systematic Review and Meta-Analysis. PLoS ONE 2016, 11, e0166915. [CrossRef]

12. Zanon-Moreno, V.; Marco-Ventura, P.; Lleo-Perez, A.; Pons-Vazquez, S.; Garcia-Medina, J.J.; Vinuesa-Silva, I.; Moreno-Nadal, M.A.; Pinazo-Duran, M.D. Oxidative stress in primary open-angle glaucoma. J. Glaucoma 2008, 17, 263-268. [CrossRef] [PubMed]

13. Li, Y.; Chen, Y.M.; Sun, M.M.; Guo, X.D.; Wang, Y.C.; Zhang, Z.Z. Inhibition on Apoptosis Induced by Elevated Hydrostatic Pressure in Retinal Ganglion Cell-5 via Laminin Upregulating beta1-integrin/Focal Adhesion Kinase/Protein Kinase B Signaling Pathway. Chin. Med. J. 2016, 129, 976-983. [CrossRef] [PubMed]

14. Nickells, R.W. Apoptosis of retinal ganglion cells in glaucoma: An update of the molecular pathways involved in cell death. Surv. Ophthalmol. 1999, 43 (Suppl. 1), S151-S161. [CrossRef]

15. Ramirez, A.I.; de Hoz, R.; Salobrar-Garcia, E.; Salazar, J.J.; Rojas, B.; Ajoy, D.; Lopez-Cuenca, I.; Rojas, P.; Trivino, A.; Ramirez, J.M. The Role of Microglia in Retinal Neurodegeneration: Alzheimer's Disease, Parkinson, and Glaucoma. Front. Aging Neurosci. 2017, 9, 214. [CrossRef]

16. Halliwell, B. Free radicals, antioxidants, and human disease: Curiosity, cause, or consequence? Lancet 1994, 344, 721-724. [CrossRef]

17. Fang, Y.Z.; Yang, S.; Wu, G. Free radicals, antioxidants, and nutrition. Nutrition 2002, 18, 872-879. [CrossRef]

18. Uttara, B.; Singh, A.V.; Zamboni, P.; Mahajan, R.T. Oxidative stress and neurodegenerative diseases: A review of upstream and downstream antioxidant therapeutic options. Curr. Neuropharmacol. 2009, 7, 65-74. [CrossRef] [PubMed]

19. Ferreira, S.M.; Lerner, S.F.; Brunzini, R.; Evelson, P.A.; Llesuy, S.F. Oxidative stress markers in aqueous humor of glaucoma patients. Am. J. Ophthalmol. 2004, 137, 62-69. [CrossRef]

20. Babizhayev, M.A. Biomarkers and special features of oxidative stress in the anterior segment of the eye linked to lens cataract and the trabecular meshwork injury in primary open-angle glaucoma: Challenges of dual combination therapy with $\mathrm{N}$-acetylcarnosine lubricant eye drops and oral formulation of nonhydrolyzed carnosine. Fundam. Clin. Pharmacol. 2012, 26, 86-117. [CrossRef]

21. McElnea, E.M.; Quill, B.; Docherty, N.G.; Irnaten, M.; Siah, W.F.; Clark, A.F.; O’Brien, C.J.; Wallace, D.M. Oxidative stress, mitochondrial dysfunction and calcium overload in human lamina cribrosa cells from glaucoma donors. Mol. Vis. 2011, 17, 1182-1191. [PubMed]

22. Engin, K.N.; Engin, G.; Kucuksahin, H.; Oncu, M.; Engin, G.; Guvener, B. Clinical evaluation of the neuroprotective effect of alpha-tocopherol against glaucomatous damage. Eur. J. Ophthalmol. 2007, 17, 528-533. [CrossRef] [PubMed]

23. Koliakos, G.G.; Befani, C.D.; Mikropoulos, D.; Ziakas, N.G.; Konstas, A.G. Prooxidant-antioxidant balance, peroxide and catalase activity in the aqueous humour and serum of patients with exfoliation syndrome or exfoliative glaucoma. Graefes. Arch. Clin. Exp. Ophthalmol. 2008, 246, 1477-1483. [CrossRef]

24. Abu-Amero, K.K.; Kondkar, A.A.; Mousa, A.; Osman, E.A.; Al-Obeidan, S.A. Analysis of catalase SNP rs1001179 in Saudi patients with primary open angle glaucoma. Ophthalmic Genet. 2013, 34, 223-228. [CrossRef]

25. Goth, L.; Nagy, T.; Kosa, Z.; Fejes, Z.; Bhattoa, H.P.; Paragh, G.; Kaplar, M. Effects of rs769217 and rs1001179 polymorphisms of catalase gene on blood catalase, carbohydrate and lipid biomarkers in diabetes mellitus. Free Radic. Res. 2012, 46, $1249-1257$. [CrossRef]

26. Casp, C.B.; She, J.X.; McCormack, W.T. Genetic association of the catalase gene (CAT) with vitiligo susceptibility. Pigment. Cell Res. 2002, 15, 62-66. [CrossRef] [PubMed]

27. Cherry, J.D.; Olschowka, J.A.; O’Banion, M.K. Neuroinflammation and M2 microglia: The good, the bad, and the inflamed. J. Neuroinflamm. 2014, 11, 98. [CrossRef] [PubMed]

28. Cuenca, N.; Fernandez-Sanchez, L.; Campello, L.; Maneu, V.; De la Villa, P.; Lax, P.; Pinilla, I. Cellular responses following retinal injuries and therapeutic approaches for neurodegenerative diseases. Prog. Retin. Eye Res. 2014, 43, 17-75. [CrossRef] [PubMed] 
29. Varnum, M.M.; Ikezu, T. The classification of microglial activation phenotypes on neurodegeneration and regeneration in Alzheimer's disease brain. Arch. Immunol. Ther. Exp. 2012, 60, 251-266. [CrossRef] [PubMed]

30. Gonzalez, H.; Elgueta, D.; Montoya, A.; Pacheco, R. Neuroimmune regulation of microglial activity involved in neuroinflammation and neurodegenerative diseases. J. Neuroimmunol. 2014, 274, 1-13. [CrossRef]

31. Jones, E.V.; Bouvier, D.S. Astrocyte-secreted matricellular proteins in CNS remodelling during development and disease. Neural Plast. 2014, 2014, 321209. [CrossRef]

32. Neufeld, A.H.; Hernandez, M.R.; Gonzalez, M. Nitric oxide synthase in the human glaucomatous optic nerve head. Arch. Ophthalmol. 1997, 115, 497-503. [CrossRef] [PubMed]

33. Shareef, S.; Sawada, A.; Neufeld, A.H. Isoforms of nitric oxide synthase in the optic nerves of rat eyes with chronic moderately elevated intraocular pressure. Investig. Ophthalmol. Vis. Sci. 1999, 40, 2884-2891.

34. Tezel, G.; Li, L.Y.; Patil, R.V.; Wax, M.B. TNF-alpha and TNF-alpha receptor-1 in the retina of normal and glaucomatous eyes. Investig. Ophthalmol. Vis. Sci. 2001, 42, 1787-1794.

35. Nakazawa, T.; Nakazawa, C.; Matsubara, A.; Noda, K.; Hisatomi, T.; She, H.; Michaud, N.; Hafezi-Moghadam, A.; Miller, J.W.; Benowitz, L.I. Tumor necrosis factor-alpha mediates oligodendrocyte death and delayed retinal ganglion cell loss in a mouse model of glaucoma. J. Neurosci. 2006, 26, 12633-12641. [CrossRef] [PubMed]

36. Vidal, L.; Diaz, F.; Villena, A.; Moreno, M.; Campos, J.G.; de Vargas, I.P. Nitric oxide synthase in retina and optic nerve head of rat with increased intraocular pressure and effect of timolol. Brain Res. Bull. 2006, 70, 406-413. [CrossRef] [PubMed]

37. Madeira, M.H.; Boia, R.; Santos, P.F.; Ambrosio, A.F.; Santiago, A.R. Contribution of microglia-mediated neuroinflammation to retinal degenerative diseases. Mediat. Inflamm. 2015, 2015, 673090. [CrossRef] [PubMed]

38. Williams, E.A.; McGuone, D.; Frosch, M.P.; Hyman, B.T.; Laver, N.; Stemmer-Rachamimov, A. Absence of Alzheimer Disease Neuropathologic Changes in Eyes of Subjects With Alzheimer Disease. J. Neuropathol. Exp. Neurol. 2017, 76, 376-383. [CrossRef] [PubMed]

39. Dinarello, C.A. A clinical perspective of IL-1beta as the gatekeeper of inflammation. Eur. J. Immunol. 2011, $41,1203-1217$. [CrossRef]

40. Dinarello, C.A. IL-1: Discoveries, controversies and future directions. Eur. J. Immunol. 2010, 40, 599-606. [CrossRef]

41. Wang, N.; Chintala, S.K.; Fini, M.E.; Schuman, J.S. Activation of a tissue-specific stress response in the aqueous outflow pathway of the eye defines the glaucoma disease phenotype. Nat. Med. 2001, 7, 304-309. [CrossRef] [PubMed]

42. Wu, C.L.; Yang, Y.T.; Wang, Y.H.; Lin, T.Y.; Lin, I.C.; Sung, C.W. Association of interleukin-6 gene polymorphisms and glaucoma: Systematic review and meta-analysis. Eur. J. Ophthalmol. 2020, 1120672120940198. [CrossRef] [PubMed]

43. Abu-Amero, K.K.; Azad, T.A.; Mousa, A.; Osman, E.A.; Sultan, T.; Al-Obeidan, S.A. A catalase promoter variant rs1001179 is associated with visual acuity but not with primary angle closure glaucoma in Saudi patients. BMC Med. Genet. 2013, 14, 84. [CrossRef] [PubMed]

44. Izzotti, A.; Sacca, S.C. Glutathione S-transferase M1 and its implications in glaucoma pathogenesis: A controversial matter. Exp. Eye Res. 2004, 79, 141-142; author reply 143. [CrossRef] [PubMed]

45. Huang, W.; Wang, W.; Zhou, M.; Chen, S.; Zhang, X. Association of glutathione S-transferase polymorphisms (GSTM1 and GSTT1) with primary open-angle glaucoma: An evidence-based meta-analysis. Gene 2013, 526, 80-86. [CrossRef] [PubMed]

46. European Glaucoma Society Terminology and Guidelines for Glaucoma. Classification and terminology Supported by the EGS Foundation: Part 1: Foreword; Introduction; Glossary; Chapter 2 Classification and Terminology; 4th Edition-Chapter 2. Br. J. Ophthalmol. 2017, 101, 73-127. [CrossRef]

47. European Glaucoma Society Terminology and Guidelines for Glaucoma. Treatment principles and options Supported by the EGS Foundation: Part 1: Foreword; Introduction; Glossary; Chapter 3 Treatment principles and options; 4th Edition-Chapter 3. Br. J. Ophthalmol. 2017, 101, 130-195. [CrossRef]

48. Redensek, S.; Flisar, D.; Kojovic, M.; Kramberger, M.G.; Georgiev, D.; Pirtosek, Z.; Trost, M.; Dolzan, V. Genetic variability of inflammation and oxidative stress genes does not play a major role in the occurrence of adverse events of dopaminergic treatment in Parkinson's disease. J. Neuroinflamm. 2019, 16, 50. [CrossRef] [PubMed]

49. Chen, C.L.; Liu, Q.; Relling, M.V. Simultaneous characterization of glutathione S-transferase M1 and T1 polymorphisms by polymerase chain reaction in American whites and blacks. Pharmacogenetics 1996, 6, 187-191. [CrossRef]

50. Dupont, W.D.; Plummer, W.D., Jr. Power and sample size calculations. A review and computer program. Control. Clin. Trials 1990, 11, 116-128. [CrossRef]

51. Stamenkovic, M.; Lukic, V.; Suvakov, S.; Simic, T.; Sencanic, I.; Pljesa-Ercegovac, M.; Jaksic, V.; Babovic, S.; Matic, M.; Radosavljevic, A.; et al. GSTM1-null and GSTT1-active genotypes as risk determinants of primary open angle glaucoma among smokers. Int. J. Ophthalmol. 2018, 11, 1514-1520. [CrossRef]

52. Rocha, A.V.; Talbot, T.; Magalhaes da Silva, T.; Almeida, M.C.; Menezes, C.A.; Di Pietro, G.; Rios-Santos, F. Is the GSTM1 null polymorphism a risk factor in primary open angle glaucoma? Mol. Vis. 2011, 17, 1679-1686.

53. Safa, F.K.; Shahsavari, G.; Abyaneh, R.Z. Glutathione s-transferase M1 and T1 genetic polymorphisms in Iranian patients with glaucoma. Iran. J. Basic Med. Sci. 2014, 17, 332-336.

54. Juronen, E.; Tasa, G.; Veromann, S.; Parts, L.; Tiidla, A.; Pulges, R.; Panov, A.; Soovere, L.; Koka, K.; Mikelsaar, A.V. Polymorphic glutathione S-transferase M1 is a risk factor of primary open-angle glaucoma among Estonians. Exp. Eye Res. 2000, 71, 447-452. [CrossRef] [PubMed] 
55. Unal, M.; Guven, M.; Devranoglu, K.; Ozaydin, A.; Batar, B.; Tamcelik, N.; Gorgun, E.E.; Ucar, D.; Sarici, A. Glutathione S transferase M1 and T1 genetic polymorphisms are related to the risk of primary open-angle glaucoma: A study in a Turkish population. Br. J. Ophthalmol. 2007, 91, 527-530. [CrossRef] [PubMed]

56. Jansson, M.; Rada, A.; Tomic, L.; Larsson, L.I.; Wadelius, C. Analysis of the Glutathione S-transferase M1 gene using pyrosequencing and multiplex PCR-no evidence of association to glaucoma. Exp. Eye Res. 2003, 77, 239-243. [CrossRef]

57. Malik, M.A.; Gupta, V.; Shukla, S.; Kaur, J. Glutathione S-transferase (GSTM1, GSTT1) polymorphisms and JOAG susceptibility: A case control study and meta-analysis in glaucoma. Gene 2017, 628, 246-252. [CrossRef] [PubMed]

58. Fan, B.J.; Liu, K.; Wang, D.Y.; Tham, C.C.; Tam, P.O.; Lam, D.S.; Pang, C.P. Association of polymorphisms of tumor necrosis factor and tumor protein p53 with primary open-angle glaucoma. Investig. Ophthalmol. Vis. Sci. 2010, 51, 4110-4116. [CrossRef]

59. Ravn-Haren, G.; Olsen, A.; Tjonneland, A.; Dragsted, L.O.; Nexo, B.A.; Wallin, H.; Overvad, K.; Raaschou-Nielsen, O.; Vogel, U. Associations between GPX1 Pro198Leu polymorphism, erythrocyte GPX activity, alcohol consumption and breast cancer risk in a prospective cohort study. Carcinogenesis 2006, 27, 820-825. [CrossRef]

60. Forsberg, L.; Lyrenas, L.; de Faire, U.; Morgenstern, R. A common functional C-T substitution polymorphism in the promoter region of the human catalase gene influences transcription factor binding, reporter gene transcription and is correlated to blood catalase levels. Free Radic. Biol. Med. 2001, 30, 500-505. [CrossRef]

61. Da Rocha, T.J.; Silva Alves, M.; Guisso, C.C.; de Andrade, F.M.; Camozzato, A.; de Oliveira, A.A.; Fiegenbaum, M. Association of GPX1 and GPX4 polymorphisms with episodic memory and Alzheimer's disease. Neurosci. Lett. 2018, 666, 32-37. [CrossRef] [PubMed]

62. Gong, B.; Shi, Y.; Qu, C.; Ye, Z.; Yin, Y.; Tan, C.; Shuai, P.; Li, J.; Guo, X.; Cheng, Y.; et al. Association of catalase polymorphisms with primary open-angle glaucoma in a Chinese population. Ophthalmic Genet. 2018, 39, 35-40. [CrossRef]

63. Zhou, Y.; Shuai, P.; Li, X.; Liu, X.; Wang, J.; Yang, Y.; Hao, F.; Lin, H.; Zhang, D.; Gong, B. Association of SOD2 polymorphisms with primary open angle glaucoma in a Chinese population. Ophthalmic Genet. 2015, 36, 43-49. [CrossRef]

64. Markiewicz, L.; Pytel, D.; Mucha, B.; Szymanek, K.; Szaflik, J.; Szaflik, J.P.; Majsterek, I. Altered Expression Levels of MMP1, MMP9, MMP12, TIMP1, and IL-1beta as a Risk Factor for the Elevated IOP and Optic Nerve Head Damage in the Primary Open-Angle Glaucoma Patients. BioMed Res. Int. 2015, 2015, 812503. [CrossRef]

65. Chi, W.; Li, F.; Chen, H.; Wang, Y.; Zhu, Y.; Yang, X.; Zhu, J.; Wu, F.; Ouyang, H.; Ge, J.; et al. Caspase-8 promotes NLRP1/NLRP3 inflammasome activation and IL-1beta production in acute glaucoma. Proc. Natl. Acad. Sci. USA 2014, 111, 11181-11186. [CrossRef]

66. Oliveira, M.B.; de Vasconcellos, J.P.C.; Ananina, G.; Costa, V.P.; de Melo, M.B. Association between IL1A and IL1B polymorphisms and primary open angle glaucoma in a Brazilian population. Exp. Biol. Med. 2018, 243, 1083-1091. [CrossRef] [PubMed]

67. Markiewicz, L.; Majsterek, I.; Przybylowska, K.; Dziki, L.; Waszczyk, M.; Gacek, M.; Kaminska, A.; Szaflik, J.; Szaflik, J.P. Gene polymorphisms of the MMP1, MMP9, MMP12, IL-1beta and TIMP1 and the risk of primary open-angle glaucoma. Acta Ophthalmol. 2013, 91, e516-e523. [CrossRef] [PubMed]

68. Lin, H.J.; Tsai, S.C.; Tsai, F.J.; Chen, W.C.; Tsai, J.J.; Hsu, C.D. Association of interleukin 1beta and receptor antagonist gene polymorphisms with primary open-angle glaucoma. Ophthalmologica 2003, 217, 358-364. [CrossRef]

69. Wang, C.Y.; Shen, Y.C.; Su, C.H.; Lo, F.Y.; Lee, S.H.; Tsai, H.Y.; Fan, S.S. Investigation of the association between interleukin-1beta polymorphism and normal tension glaucoma. Mol. Vis. 2007, 13, 719-723.

70. How, A.C.; Aung, T.; Chew, X.; Yong, V.H.; Lim, M.C.; Lee, K.Y.; Toh, J.Y.; Li, Y.; Liu, J.; Vithana, E.N. Lack of association between interleukin-1 gene cluster polymorphisms and glaucoma in Chinese subjects. Investig. Ophthalmol. Vis. Sci. 2007, 48, 2123-2126. [CrossRef]

71. Mookherjee, S.; Banerjee, D.; Chakraborty, S.; Mukhopadhyay, I.; Sen, A.; Ray, K. Evaluation of the IL1 Gene Cluster Single Nucleotide Polymorphisms in Primary Open-Angle Glaucoma Pathogenesis. Genet. Test. Mol. Biomark. 2016, 20, 633-636. [CrossRef] [PubMed]

72. Li, J.; Feng, Y.; Sung, M.S.; Lee, T.H.; Park, S.W. Association of Interleukin-1 gene clusters polymorphisms with primary open-angle glaucoma: A meta-analysis. BMC Ophthalmol. 2017, 17, 218. [CrossRef] [PubMed]

73. Zimmermann, C.; Weger, M.; Faschinger, C.; Renner, W.; Mossbock, G. Role of interleukin 6-174G $>$ C polymorphism in primary open-angle glaucoma. Eur. J. Ophthalmol. 2013, 23, 183-186. [CrossRef]

74. Ye, H.; Li, X.; Wang, L.; Liao, Q.; Xu, L.; Huang, Y.; Xu, L.; Xu, X.; Chen, C.; Wu, H.; et al. Genetic associations with coronary heart disease: Meta-analyses of 12 candidate genetic variants. Gene 2013, 531, 71-77. [CrossRef]

75. Rosenberger, A.; Illig, T.; Korb, K.; Klopp, N.; Zietemann, V.; Wolke, G.; Meese, E.; Sybrecht, G.; Kronenberg, F.; Cebulla, M.; et al. Do genetic factors protect for early onset lung cancer? A case control study before the age of 50 years. BMC Cancer 2008, 8, 60 . [CrossRef]

76. Malinowska, K.; Kowalski, M.; Szaflik, J.; Szaflik, J.P.; Majsterek, I. The role of Cat-262C/T, GPX1 Pro198Leu and Sod1+35A/C gene polymorphisms in a development of primary open-angle glaucoma in a Polish population. Pol. J. Pathol. 2016, 67, 404-410. [CrossRef] [PubMed]

77. Wolfs, R.C.; Klaver, C.C.; Ramrattan, R.S.; van Duijn, C.M.; Hofman, A.; de Jong, P.T. Genetic risk of primary open-angle glaucoma. Population-based familial aggregation study. Arch. Ophthalmol. 1998, 116, 1640-1645. [CrossRef] [PubMed]

78. Cheema, A.; Chang, R.T.; Shrivastava, A.; Singh, K. Update on the Medical Treatment of Primary Open-Angle Glaucoma. Asia Pac. J. Ophthalmol. 2016, 5, 51-58. [CrossRef] 
79. Springelkamp, H.; Iglesias, A.I.; Mishra, A.; Hohn, R.; Wojciechowski, R.; Khawaja, A.P.; Nag, A.; Wang, Y.X.; Wang, J.J.; Cuellar-Partida, G.; et al. New insights into the genetics of primary open-angle glaucoma based on meta-analyses of intraocular pressure and optic disc characteristics. Hum. Mol. Genet. 2017, 26, 438-453. [CrossRef] [PubMed]

80. Choquet, H.; Wiggs, J.L.; Khawaja, A.P. Clinical implications of recent advances in primary open-angle glaucoma genetics. Eye 2020, 34, 29-39. [CrossRef] [PubMed]

81. Khawaja, A.P.; Cooke Bailey, J.N.; Wareham, N.J.; Scott, R.A.; Simcoe, M.; Igo, R.P., Jr.; Song, Y.E.; Wojciechowski, R.; Cheng, C.Y.; Khaw, P.T.; et al. Genome-wide analyses identify 68 new loci associated with intraocular pressure and improve risk prediction for primary open-angle glaucoma. Nat. Genet. 2018, 50, 778-782. [CrossRef]

82. Gordon, M.O.; Beiser, J.A.; Brandt, J.D.; Heuer, D.K.; Higginbotham, E.J.; Johnson, C.A.; Keltner, J.L.; Miller, J.P.; Parrish, R.K., 2nd; Wilson, M.R.; et al. The Ocular Hypertension Treatment Study: Baseline factors that predict the onset of primary open-angle glaucoma. Arch. Ophthalmol. 2002, 120, 714-720; discussion 829-830. [CrossRef] [PubMed]

83. Leske, M.C.; Heijl, A.; Hyman, L.; Bengtsson, B.; Dong, L.; Yang, Z. Predictors of long-term progression in the early manifest glaucoma trial. Ophthalmology 2007, 114, 1965-1972. [CrossRef] [PubMed]

84. Medeiros, F.A.; Meira-Freitas, D.; Lisboa, R.; Kuang, T.M.; Zangwill, L.M.; Weinreb, R.N. Corneal Hysteresis as a Risk Factor for Glaucoma Progression: A Prospective Longitudinal Study. Ophthalmology 2013, 120, 1533-1540. [CrossRef]

85. Mitchell, P.; Lee, A.J.; Rochtchina, E.; Wang, J.J. Open-angle glaucoma and systemic hypertension: The blue mountains eye study. J. Glaucoma 2004, 13, 319-326. [CrossRef] [PubMed]

86. Klein, B.E.; Klein, R.; Knudtson, M.D. Intraocular pressure and systemic blood pressure: Longitudinal perspective: The Beaver Dam Eye Study. Br. J. Ophthalmol. 2005, 89, 284-287. [CrossRef]

87. Hayreh, S.S.; Podhajsky, P.; Zimmerman, M.B. Ocular and optic nerve head ischemic disorders and hearing loss. Am. J. Ophthalmol. 1999, 128, 606-611. [CrossRef]

88. Hayreh, S.S.; Podhajsky, P.; Zimmerman, M.B. Beta-blocker eyedrops and nocturnal arterial hypotension. Am. J. Ophthalmol. 1999, 128, 301-309. [CrossRef]

89. Zhao, D.; Cho, J.; Kim, M.H.; Friedman, D.S.; Guallar, E. Diabetes, fasting glucose, and the risk of glaucoma: A meta-analysis. Ophthalmology 2015, 122, 72-78. [CrossRef]

90. Ko, F.; Boland, M.V.; Gupta, P.; Gadkaree, S.K.; Vitale, S.; Guallar, E.; Zhao, D.; Friedman, D.S. Diabetes, Triglyceride Levels, and Other Risk Factors for Glaucoma in the National Health and Nutrition Examination Survey 2005-2008. Investig. Ophthalmol. Vis. Sci. 2016, 57, 2152-2157. [CrossRef]

91. Shen, L.; Walter, S.; Melles, R.B.; Glymour, M.M.; Jorgenson, E. Diabetes Pathology and Risk of Primary Open-Angle Glaucoma: Evaluating Causal Mechanisms by Using Genetic Information. Am. J. Epidemiol. 2016, 183, 147-155. [CrossRef]

92. Song, B.J.; Aiello, L.P.; Pasquale, L.R. Presence and Risk Factors for Glaucoma in Patients with Diabetes. Curr. Diabetes Rep. 2016, 16, 124. [CrossRef]

93. Chen, Y.Y.; Hu, H.Y.; Chu, D.; Chen, H.H.; Chang, C.K.; Chou, P. Patients with Primary Open-Angle Glaucoma May Develop Ischemic Heart Disease More Often than Those without Glaucoma: An 11-Year Population-Based Cohort Study. PLoS ONE 2016, 11, e0163210. [CrossRef] [PubMed]

94. Luo, X.Y.; Dai, W.; Chee, M.L.; Tao, Y.; Chua, J.; Tan, N.Y.Q.; Tham, Y.C.; Aung, T.; Wong, T.Y.; Cheng, C.Y. Association of Diabetes With Central Corneal Thickness Among a Multiethnic Asian Population. JAMA Netw. Open 2019, 2, e186647. [CrossRef]

95. Karvonen, E.; Stoor, K.; Luodonpaa, M.; Hagg, P.; Kuoppala, J.; Lintonen, T.; Ohtonen, P.; Tuulonen, A.; Saarela, V. Prevalence of glaucoma in the Northern Finland Birth Cohort Eye Study. Acta Ophthalmol. 2019, 97, 200-207. [CrossRef]

96. Klein, B.E.; Klein, R.; Jensen, S.C. Open-angle glaucoma and older-onset diabetes. The Beaver Dam Eye Study. Ophthalmology 1994, 101, 1173-1177. [CrossRef]

97. Dielemans, I.; Vingerling, J.R.; Wolfs, R.C.; Hofman, A.; Grobbee, D.E.; de Jong, P.T. The prevalence of primary open-angle glaucoma in a population-based study in The Netherlands. The Rotterdam Study. Ophthalmology 1994, 101, 1851-1855. [CrossRef]

98. Mizzi, C.; Dalabira, E.; Kumuthini, J.; Dzimiri, N.; Balogh, I.; Basak, N.; Bohm, R.; Borg, J.; Borgiani, P.; Bozina, N.; et al. A European Spectrum of Pharmacogenomic Biomarkers: Implications for Clinical Pharmacogenomics. PLoS ONE 2016, 11, e0162866. [CrossRef] [PubMed]

99. Vidan-Jeras, B.; Jurca, B.; Dolzan, V.; Jeras, M.; Breskvar, K.; Bohinjec, M. Slovenian Caucasian Normal. In HLA 1998; Terasaki, P.I., Gjertson, D.W., Lenaxa, K.S., Eds.; American Society for Histocompatibility and Immunogenetics: Mount Laurel, NJ, USA, 1998; pp. $180-181$.

100. Ramdas, W.D.; Schouten, J.; Webers, C.A.B. The Effect of Vitamins on Glaucoma: A Systematic Review and Meta-Analysis. Nutrients 2018, 10, 359. [CrossRef]

101. Pinazo-Duran, M.D.; Shoaie-Nia, K.; Zanon-Moreno, V.; Sanz-Gonzalez, S.M.; Del Castillo, J.B.; Garcia-Medina, J.J. Strategies to Reduce Oxidative Stress in Glaucoma Patients. Curr. Neuropharmacol. 2018, 16, 903-918. [CrossRef]

102. Hui, F.; Tang, J.; Williams, P.A.; McGuinness, M.B.; Hadoux, X.; Casson, R.J.; Coote, M.; Trounce, I.A.; Martin, K.R.; van Wijngaarden, P.; et al. Improvement in inner retinal function in glaucoma with nicotinamide (vitamin B3) supplementation: A crossover randomized clinical trial. Clin. Exp. Ophthalmol. 2020, 48, 903-914. [CrossRef]

103. Adornetto, A.; Russo, R.; Parisi, V. Neuroinflammation as a target for glaucoma therapy. Neural Regen. Res. 2019, 14, 391-394. [CrossRef] 
104. Ban, N.; Siegfried, C.J.; Apte, R.S. Monitoring Neurodegeneration in Glaucoma: Therapeutic Implications. Trends Mol. Med. 2018, 24, 7-17. [CrossRef]

105. Guo, X.; Namekata, K.; Kimura, A.; Harada, C.; Harada, T. ASK1 in neurodegeneration. Adv. Biol. Regul. 2017, 66, 63-71. [CrossRef]

106. Sutton, A.; Imbert, A.; Igoudjil, A.; Descatoire, V.; Cazanave, S.; Pessayre, D.; Degoul, F. The manganese superoxide dismutase Ala16Val dimorphism modulates both mitochondrial import and mRNA stability. Pharmacogenet. Genom. 2005, 15, 311-319. [CrossRef]

107. Nebert, D.W.; Vasiliou, V. Analysis of the glutathione S-transferase (GST) gene family. Hum Genom. 2004, 1, 460-464. [CrossRef] [PubMed]

108. Kutikhin, A.G.; Yuzhalin, A.E.; Volkov, A.N.; Zhivotovskiy, A.S.; Brusina, E.B. Correlation between genetic polymorphisms within IL-1B and TLR4 genes and cancer risk in a Russian population: A case-control study. Tumor Biol. 2014, 35, 4821-4830. [CrossRef]

109. Torres-Merino, S.; Moreno-Sandoval, H.N.; Thompson-Bonilla, M.D.R.; Leon, J.A.O.; Gomez-Conde, E.; Leon-Chavez, B.A.; Martinez-Fong, D.; Gonzalez-Barrios, J.A. Association Between rs3833912/rs16944 SNPs and Risk for Cerebral Palsy in Mexican Children. Mol. Neurobiol. 2018, 21, 018-1178. [CrossRef] [PubMed]

110. Lagmay, J.P.; London, W.B.; Gross, T.G.; Termuhlen, A.; Sullivan, N.; Axel, A.; Mundy, B.; Ranalli, M.; Canner, J.; McGrady, P.; et al. Prognostic significance of interleukin-6 single nucleotide polymorphism genotypes in neuroblastoma: rs1800795 (promoter) and rs8192284 (receptor). Clin. Cancer Res. 2009, 15, 5234-5239. [CrossRef] [PubMed]

111. Szkup, M.; Chelmecka, E.; Lubkowska, A.; Owczarek, A.J.; Grochans, E. The influence of the TNFalpha rs1800629 polymorphism on some inflammatory biomarkers in 45-60-year-old women with metabolic syndrome. Aging 2018, 10, 2935-2943. [CrossRef] 